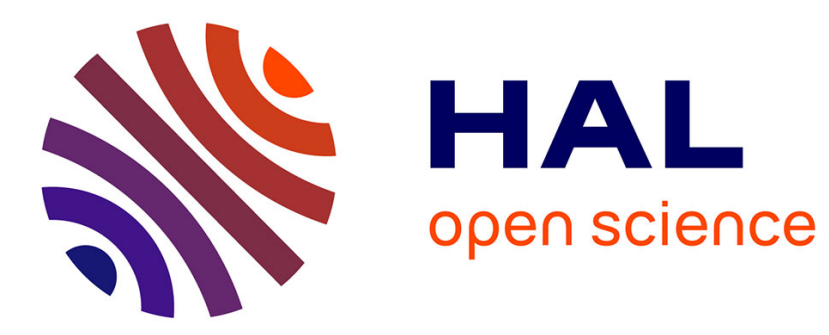

\title{
Analysis of the Anatomical Variability of Fetal Brains with Corpus Callosum Agenesis
}

Fleur Gaudfernau, Eleonore Blondiaux, Stéphanie Allassonnière

\section{To cite this version:}

Fleur Gaudfernau, Eleonore Blondiaux, Stéphanie Allassonnière. Analysis of the Anatomical Variability of Fetal Brains with Corpus Callosum Agenesis. 2022. hal-03546165

\section{HAL Id: hal-03546165 \\ https://hal.science/hal-03546165}

Preprint submitted on 28 Jan 2022

HAL is a multi-disciplinary open access archive for the deposit and dissemination of scientific research documents, whether they are published or not. The documents may come from teaching and research institutions in France or abroad, or from public or private research centers.
L'archive ouverte pluridisciplinaire HAL, est destinée au dépôt et à la diffusion de documents scientifiques de niveau recherche, publiés ou non, émanant des établissements d'enseignement et de recherche français ou étrangers, des laboratoires publics ou privés. 


\title{
PIPPI2021 - Analysis of the Anatomical Variability of Fetal Brains with Corpus Callosum Agenesis
}

Fleur Gaudfernau

fleur.gaudfernau@etu.u-paris.fr

CRC, Université de Paris, INRIA EPI HEKA, INSERM UMR 1138, Sorbonne Université

Eléonore Blondiaux

Service de Radiologie, Hôpital d'Enfants Armand-Trousseau, APHP

Stéphanie Allassonière

CRC, Université de Paris, INRIA EPI HEKA, INSERM UMR 1138, Sorbonne Université

\begin{abstract}
Corpus Callosum Agenesis (CCA), one of the most common congenital anomalies, has uncertain neurodevelopmental outcome, especially when the disease is isolated. To provide parents with informed counselling, it is crucial to identify anatomical markers linked to a predicted outcome early in pregnancy. Quantitative exploration of fetal brains with CCA is rare and has been mostly limited to the study of specific brain structures. Here, we propose a pipeline to analyse fetal brain Magnetic Resonance Imaging (MRI) that is based on diffeomorphic transformation. It consists in two steps: a semi-automatic fetal MRI preprocessing procedure and a pipeline to quantify anatomical deviations from normal development. Following MRI preprocessing, each volumetric fetal brain is compared to an age-matched healthy template brain at a global scale using registration. Deformations are parallel transported to the same space to correct age differences between fetuses. Deformation modes specific to CCA are identified using Principal Component Analysis and classification. The pipeline is tested on retrospectively selected MRIs from 38 healthy fetuses and 73 fetuses with CCA. In accordance with more local analyses, the most relevant deformation mode for classification combines well-known alterations of brains with CCA. This preliminary work is promising for the quantitative exploration of abnormal fetal brains and will be used in the future to identify anatomical features correlated to poor clinical outcome.
\end{abstract}

Keywords: Corpus Callosum Agenesis, Fetal Magnetic Resonance Imaging, Diffeomorphic Registration, Shape Analysis

\section{Introduction}

Corpus callosum agenesis (CCA) is one of the most common congenital brain anomalies, with a prevalence at birth of $0.02 \%$ (Leombroni et al., 2018). It is characterized by the total or partial absence of the largest commissure of the brain, responsible for the transmission of sensory, motor and cognitive information between hemispheres (Leombroni et al., 2018). Diagnosis is usually suspected during the second-trimester routine ultrasound, and confirmed by Magnetic Resonance Imaging (MRI) scan (Leombroni et al., 2018). In complement with genetic screening, fetal MRI is valuable to provide clinicians with additional information, 
since the presence of other anomalies is the only consensual prognosis factor for neurodevelopmental delays (Santo et al., 2012). In the presence of associated defects, accounting for $45 \%$ cases (Santo et al., 2012), the outcome is usually poor, with impairments affecting motor control, coordination and language (D'Antonio et al., 2016). Predicting the outcome is challenging in isolated CCA, where $20-30 \%$ children demonstrate a broad spectrum of cognitive deficits (Santo et al., 2012; D'Antonio et al., 2016), resulting in heterogenous medical counselling across hospitals and countries (Des Portes et al., 2018). To provide parents with informed counselling, it is crucial to identify anatomical markers linked to neurodevelopmental outcome as early as possible during pregnancy.

Quantitative in vivo analysis of fetal brains has long been limited by the scarcity of fetal MRI data and its restriction to 2D slices (Clouchoux et al., 2011). Indeed, unpredictable fetal and maternal motion make the acquisition of 3D images challenging. With the advent of fast single shot multi-slice MRI sequences, combined with postprocessing techniques, it is now possible to acquire stacks of $2 \mathrm{D}$ images with reasonable in-plane motion, perform inter-slice motion correction, and reconstruct a high resolution volumetric image of the fetal brain (Benkarim et al., 2017). Taking advantage of these recent developments, the quantitative assessment of normal and pathological brain development has attracted growing interest (Benkarim et al., 2017). However, to this day, only few studies have investigated quantitatively anatomical alterations in fetuses with CCA (Knezović et al., 2019; Tarui et al., 2018; Schwartz et al., 2021), and their focus was on specific brain structures rather than global trends. Another limitation is the difficulty to compare fetal brains of different gestational ages, since they undergo rapid and drastic changes across pregnancy (Gholipour et al., 2017).

Whole brain shape analysis can provide information about which structures are impaired along with corpus callosum. To perform such global analysis, one can think of image registration, which maps a population average brain template onto individual images in order to measure a distance from normality. In a clinical setting, functions called diffeomorphisms are an appropriate choice for computing shape changes, as they are high dimensional, topology-preserving, and sensitive to small anatomical variations. The Large Deformation Diffeomorphic Metric Mapping (LDDMM) setting (Trouvé, 1998; Christensen et al., 1996) is a powerful method for computing such functions, which are seen as geodesics on a Riemanian manifold. Diffeomorphisms can be efficiently computed through a discrete parametrization (Durrleman et al., 2012). The LDDMM framework also provides geometrical tools such as parallel transport, which enables the comparison of subjects that are at different developmental stages. Diffeomorphisms have proven useful in the quantification and classification of disorders such as Alzheimer's disease (Debavelaere et al., 2020; Qiu et al., 2008). To our knowledge, deformation models have never been applied to abnormal fetal brains.

Here, we propose to explore the anatomical variability of fetal brains diagnosed with CCA by introducing a novel shape analysis pipeline, based on diffeomorphic brain mapping and specifically tailored to the specificities of fetal MRI. Following data preprocessing, a healthy template will be registered to each subject, and age-related differences between fetuses will be corrected by transporting deformations to a common space. Deformations specific to fetal brains with CCA will be identified using Principal Component Analysis (PCA) and classification. 
This article extends our work presented at the MICCAI PIPPI workshop (Gaudfernau et al., 2021) in the following manner: we conduct an in-depth literature review of the quantitative analysis of fetal brains; we describe further the geometrical tools used in our shape analysis pipeline; and we provide a detailed description of our semi-automatic MRI preprocessing pipeline adapted to abnormal, routinely acquired fetal MRIs.

\section{Related Works}

Here, we aim at providing the reader with an overview of the methods that have enabled automatic analysis and quantification of fetal brain MRI. In Section 2.1, we address the challenges of the brain reconstruction task. In Section 2.2, we cover quantitative studies of healthy and abnormal fetal brains based on volume-reconstructed T2 MRIs. We demonstrate that while a number of automatic methods have been introduced in the literature, the lack of fully automated pipelines encompassing both reconstruction and analysis tools have limited the exploration of brain development -including in fetuses with CCA- and the reproducibility of such studies.

\subsection{Fetal brain reconstruction}

Advances in the acquisition of fetal MRI have enabled radiologists to image the fetal brain in less than a second, thus mitigating in-plane maternal and fetal movements (Gholipour et al., 2015). However, numerous difficulties remain, among which the low resolution of the acquired 2D images, inter-slice motion and bias field artifacts, the presence of maternal tissues around the fetal brain, and unknown orientation of the fetus with regard to the scanner. As the postprocessing tools for postnatal MRI cannot be transferred to fetal images, dedicated techniques have emerged, that aim at reconstructing a high resolution volumetric image from motion corrupted stacks of 2D slices acquired in orthogonal orientations (Clouchoux et al., 2011). Brain extraction, i.e. the task of delineating the fetal brain from the surrounding tissues such as the placenta, is often a prerequisite for volume reconstruction. As most studies have focused separately on either task, we shall review first brain extraction techniques, then volume reconstruction strategies. Note that segmentation techniques fall out of the scope of this paper.

\subsubsection{BRAIN EXTRACTION}

The first algorithm (Anquez et al., 2009) for brain extraction selected the mid-sagittal slice by detecting the eye with template matching, then isolated the brain on this $2 \mathrm{D}$ slice and subsequently on the $3 \mathrm{D}$ volume using a graph cut approach. It assumed low inter-slice motion, which can be unrealistic in practice. It was followed by other templatebased strategies. In (Taleb et al., 2013), each 2D slice was mapped to an age-specific template to estimate a brain mask. Quality of the 2D brain extraction was assessed by computing a similarity measure between the estimated masks. In (Tourbier et al., 2015b), after manual brain localization, several brain templates were registered to each $2 \mathrm{D}$ slice and brain extraction was performed with a global voting strategy. The robustness of these methods to pathologies is impeded by the use of a healthy template. 
Other methods favored learning-based approaches. In (Ison et al., 2012), a random forest classifier was trained to distinguish maternal from fetal tissues and the fetus orientation was recovered by estimating the position of the centroid of several tissues. (Keraudren et al., 2013) localized the fetal brain by describing candidate regions with scale and rotation invariant features and trained a Support Vector Machine (SVM) to delineate a bounding box around the brain. Then, slice-by-slice patch-based extraction was performed inside the bounding box to extract the brain (Keraudren et al., 2014). (Kainz et al., 2014) computed rotation invariant descriptors of the $3 \mathrm{D}$ volume and trained a random forest classifier to produce a probability map of brain voxels, which was refined for final brain extraction. (Alansary et al., 2015) decomposed the 2D low resolution images into superpixels, computed descriptors for each superpixel, and trained a random forest to generate a probability map, which was refined by another random forest. More recently, deep learning methods have emerged. (Rajchl et al., 2016) employed a Convolutional Neural Network (CNN) trained with weak annotations by non-expert raters. (Khalili et al., 2017) predicted brain masks from input 2D patches with a a multi-scale CNN. (Salehi et al., 2018) reused a CNN called U-net, which had showed high performance on 3D adult brain extraction, and adapted it to 2D fetal MRI slices. Finally, (Ebner et al., 2020) introduced a two-step procedure whereby a first CNN performs coarse localization of the brain region, followed by fine brain delineation by a second CNN.

\subsubsection{Volume RECONSTRUCTION}

Typical volume reconstruction techniques comprise two main steps (Clouchoux et al., 2011). First, motion correction is performed through Slice-to-Volume (SVR) rigid registration in order to correct the discrepancy between the positions of the 2D slices. The best alignment between each image and an arbitrarily chosen target slice is achieved by minimizing a similarity metric (e.g. Normalized Mutual Information, Cross Correlation, Mean Square Intensity) with gradient-descent optimization. Additional steps may include outlier rejection to identify and exclude highly motion corrupted slices. Then, Super Resolution Reconstruction (SRR) restores a 3D volume either by performing scattered data interpolation or solving an inverse problem with spatial regularization.

The majority of reconstruction algorithms perform SVR registration and SRR in an iterative manner. Pioneer strategies iterated between SVR registration and scattered data interpolation (Rousseau et al., 2006; Jiang et al., 2007). As an alternative to SVR, (Kim et al., 2009) introduced a motion correction technique in which slices are collectively aligned by matching their intersections, followed by Gaussian-weighted interpolation. The following algorithms formulated the SRR inverse problem in a variational framework. (Rousseau et al., 2010) expressed the SRR task as an inverse problem solved with Total Variation regularization. Similarly, (Gholipour et al., 2010) formulated the SRR task as a maximum likelihood error norm minimization problem and performed outlier rejection to reduce the weight of motion-corrupted voxels and slices. Building on this idea, (Deprez et al., 2012) iterated between a SVR approach similar to that of (Rousseau et al., 2006) and a Bayesian formulation of the SRR problem with a complete outlier rejection scheme based on the Expectation-Minimization algorithm. A GPU support of this algorithm was developed by (Kainz et al., 2015), complemented with an automatic detection of the slice with least motion 
to serve as reference during SVR. (Tourbier et al., 2015a) further improved the SRR step by minimizing the Total Variation energy with convex optimization. (Ebner et al., 2020) estimated an initial high resolution volume using scattered data approximation, followed by a classical iterative registration-reconstruction procedure with robust outlier detection. Recent years have also witnessed the emergence of machine learning approaches. (Hou et al., 2017) trained a CNN to predict a good initial slice alignment prior to the SVR registration, which proved robust for scans with extreme slice motion corruption. (McDonagh et al., 2017) used a 3D CNN to upsample each low-resolution stack during the SRR step prior to SVR motion correction.

\subsubsection{Limitations OF EXISTING VOLUME RECONSTRUCTION METHODS}

Despite the number of existing reconstruction approaches, the volumetric analysis of fetal brains is still hampered by several limitations. First, most studies do not propose a fully automated reconstruction pipeline, i.e. comprising brain localization, extraction and reorientation along with motion correction and reconstruction, despite these steps often being necessary to perform inter-subject comparisons in a quantitative manner. As a matter of fact, a number of approaches (Kim et al., 2009; Rousseau et al., 2010; Gholipour et al., 2010; McDonagh et al., 2017) perform volume reconstruction without prior or subsequent brain extraction, despite its potential to improve the reconstruction outcome (Tourbier et al., 2015b). Others (Rousseau et al., 2006; Jiang et al., 2007; Deprez et al., 2012; Tourbier et al., 2015a) delineate the brain in a semi-automatic manner, which is both resource and time consuming. Similarly, reorientation of the brain in the canonical space is either overlooked (Rousseau et al., 2006; Jiang et al., 2007; Rousseau et al., 2010; Gholipour et al., 2010) or performed manually (Rousseau et al., 2012; Tourbier et al., 2015a).

To this day, few fully automated pipelines have been proposed. The open-source toolkit of (Kainz et al., 2015) does not include extraction nor reorientation of the fetal brain. In the Baby Brain Toolkit (Rousseau et al., 2012), that reconstructs fetal brains with non local denoising, optional brain extraction is performed manually, while reorientation is carried out by positioning landmarks on the reconstructed brain. The first complete pipeline (Tourbier et al., 2017) includes template-based brain localisation and extraction, along with reorientation in the standard radiological anatomical planes. Finally, a state-ofthe-art reconstruction pipeline (Ebner et al., 2020) was recently introduced, offering brain localization and extraction with two CNNs, iterative SVR and outlier-robust SRR, and registration of the reconstructed brain to a template space.

Another limitation lies in the performance evaluation of these algorithms, complicated by the absence of ground truth and limited amount of data. Most approaches were evaluated on less than twenty -exclusively healthy- fetuses, preventing a complete demonstration of robustness. Though the pipeline of (Tourbier et al., 2017) was tested on 5 abnormal fetuses, these were only mildly pathological. Only the most recent study (Ebner et al., 2020) conducted experiments on routinely acquired images of 37 healthy fetuses and 32 subjects with spina bifida, but this cohort might be insufficient to represent the variety of pathological anatomies that can be encountered in fetal brain MRI. 


\subsection{Quantitative analysis of fetal brains}

In the last decade, the postprocessing techniques developed for fetal MRI have made possible to study quantitatively the growth of brain tissues. Our aim here is provide the reader with an overview of the techniques employed, the structures of interest and the limitations of the studies that have quantified the evolution of healthy and abnormal brains in volumereconstructed fetal MRIs. For extensive reviews about the findings of such studies, see Benkarim et al. (2017); Studholme and Rousseau (2013); Rajagopalan et al. (2021); Biegon and Hoffmann (2014); Rousseau et al. (2016).

\subsubsection{Healthy fetal BRains}

The analysis of healthy fetal brains has mostly focused on measurements of specific brain volumes, quantification of the cortical folding process, and longitudinal analyses of brain evolution throughout pregnancy.

To assess the dynamics of global or regional brain changes, volumetric studies classiquely perform manual or automatic brain segmentation in order to compute tissue volumes, which are then incorporated in temporal models. (Gholipour et al., 2011) performed linear and quadratic fits of the total brain volume with age. The relationship between gestational age and several tissue volumes such as gray matter, white matter, and ventricles were tested using linear, quadratic and exponential models (Scott et al., 2011a; Corbett-Detig et al., 2010). Relying on a cohort of 166 fetuses, (Andescavage et al., 2016) presented normative growth curves of several structures using quantile regression and compared hemispheric growth rates. Some studies focused on a single structure such as the hippocampus (Jacob et al., 2011) and the cerebellum (Scott et al., 2011b), which were manually segmented to carry out inter-hemispheric volume comparisons and statistical modelling with age.

The gyrification process in the fetal brain has been widely explored using quantitative measures of the cortical surface curvature at the global level (Hu et al., 2011; Clouchoux et al., 2011; Wright et al., 2014; Wu et al., 2015) and vertex level (Habas et al., 2011). These curvature analyses usually rely on segmentation and reconstruction of the cortical plate, derivation of cortical folding measures, and temporal modelling. (Hu et al., 2011) computed global and regional measures of the gyral and sulcal surfaces, whose evolution was tested against time and compared between brain lobes. (Habas et al., 2011) computed a vertexwise mean curvature index and showed a linear evolution of cortical folding between 20 and 28 gestational weeks (GW). (Clouchoux et al., 2011) introduced an algorithm to establish individual probability maps of sulci location and showed an acceleration of the gyrification process during the third trimester. Several cortical folding measures were found to predict accurately gestational age using linear (Wu et al., 2015) and Gompertz models (Wright et al., 2014). The cortical folding process was also explored using tensor-based morphometry, which computes the deformation of images with regard to a reference anatomy in order to capture local shape differences (Rajagopalan et al., 2011, 2012). This enabled to compute growth maps of the cerebrum and the cortical plate and study the directionality of volume changes. This work was extended by (Pontabry et al., 2015), which performed feature selection to extract sparse local deformation fields that provide information about which zones of the cortical plate undergo major changes across gestation. 
Further, several studies have modelled the evolution of normal brain anatomy across pregnancy by providing intensity brain templates and tissue probability maps for each gestational age (Habas et al., 2010; Wright et al., 2015; Gholipour et al., 2017; Li et al., 2021). These models, termed atlases, have a large scope of application as they provide an insight into healthy brain growth and may serve as reference to segment new subjects and characterize abnormal brains. They differ in several regards: the number of subjects included (from 20 to 212); the considered age range; the construction methology; and the regions of interest (cortical surface (Wright et al., 2015) or whole-brain templates (Habas et al., 2010; Gholipour et al., 2017; Li et al., 2021)).

\subsubsection{ABNORMAL FETAL BRAINS}

The quantitative analysis of abnormal fetal brains is still in its infancy. So far, it has been restricted to few abnormalities and pathologies, including mainly ventriculomegaly, Chronic Heart Disease, and spina bifida.

As in studies focusing on healthy brains, volumetric analyses are predominant. Following manual or semi-automatic brain segmentation, regression models were employed to compare the temporal evolution of several tissue volumes between fetuses with CHD and healthy controls (Clouchoux et al., 2012; Rollins et al., 2020) and between two subtypes of CHD (Rajagopalan et al., 2018). (Gholipour et al., 2012) developed a multi-atlas based segmentation method robust to ventricular abnormalities and compared the reliability of ventriculomegaly diagnosis using a measure of ventricular volume versus atrial diameter. (Kyriakopoulou et al., 2013; Scott et al., 2012) extracted the ventricles and several brain structures to perform volumetric comparisons between fetuses with ventriculomegaly and normal fetuses, with contrasted results.

Curvature analyses were performed to highlight delays in gyrification dynamics in populations of fetuses with CHD and ventriculomegaly. (Clouchoux et al., 2012) reconstructed the surface of the cortical gray matter in fetuses with a severe form of CHD. Linear regressions were used to compare the changes in curvature measures between controls and subjects. (Scott et al., 2012) compared curvatures of the ventricles and cortical plate between healthy fetuses and fetuses with ventriculomegaly. In a similar study, (Benkarim et al., 2018) identified regions of delayed cortical folding related to ventriculomegaly. They later adopted a less typical approach based on manifold learning in order to find associations between abnormal growths of the cortical and ventricular surfaces (Benkarim et al., 2020). The common underlying representation of vertices belonging to both anatomies provided information about which cortical areas are related to specific dilated ventricular regions.

Longitudinal analyses have explored the anatomical characteristics of fetal brains with spina bifida. (Payette et al., 2019) investigated the change of ventricle shape in fetuses that underwent prenatal surgery. Using deformation-based morphometry, post-operation ventricles were registered to the pre-operation ones, and statistical analysis of the deformation maps was performed. (Fidon et al., 2021b) created a week-by-week spatiotemporal atlas of fetal brains with spina bifida using 90 MRIs from 37 subjects acquired between 21 and 34 GW. The fetal atlas was parcellated in a semi-automated manner. 


\subsubsection{Fetal Brains with Corpus Callosum Agenesis}

The brain anatomy of individuals with CCA has been mostly investigated using either in utero 2D MRI and ultrasound data, or postnatal images (Nakata et al., 2009; Bénézit et al., 2015). Quantitative studies of fetal brains with CCA are listed in Table 1. To the best of our knowledge, quantitative analyses of volumetric fetal MRI have been attempted in only three studies (Knezović et al., 2019; Tarui et al., 2018; Schwartz et al., 2021), with a focus on specific brain structures. (Knezović et al., 2019) measured the hippocampal volume by means of manual delineation on 3D reconstructed MRIs from 39 healthy fetuses and 46 fetuses with CCA. Statistical testing showed reduced hippocampal volume in impaired fetuses. (Tarui et al., 2018) investigated cortical folding in 17 controls and 7 fetuses with CCA. Following reconstruction of the surface of the inner cortical plate, sulcal developing basins were identified and matched to healthy sulcal templates, revealing abnormalities in sulcal positions. (Schwartz et al., 2021) also explored the cortical morphology of 46 fetuses with CAA and 22 healthy fetuses. Measures of surface area, gyrification, thickness of the cerebral wall and cortical asymmetry were computed in several cerebral areas to assess differences between groups, showing reduced cerebral wall thickness and structural asymmetry in several brain regions in fetuses with CCA.

Of note, two studies (Kasprian et al., 2013; Jakab et al., 2015) have analysed Diffusion Tensor Imaging (DTI) data, which maps the diffusion of water molecules within brain tissues and allows to reconstruct the 3D route of white matter fibers. (Kasprian et al., 2013) analysed DTI data from 20 fetuses with CCA and 20 age-matched healthy fetuses between 20 and $37 \mathrm{GW}$. Trajectories of abnormal white matter tracts, namely the Probst bundles and sigmoid bundles, were visualized in subjects with complete and partial CCA, respectively. Alterations in somatosensory and motor pathways were also highlighted. Using the same method, (Jakab et al., 2015) further showed that fetuses with CCA have aberrant organization of the brain connectome, notably decreased interhemispheric structural connectivity and increased connectivity in intrahemispheric alternative information pathways.

Table 1: Papers performing quantitative analysis of fetal brains with corpus callosum agenesis. Data: type of data used (either T2 MRI or DTI). N: number of subjects included in the study (subjects with CCA / healthy controls)

\begin{tabular}{|c|c|c|c|c|}
\hline Reference & Data & $\mathrm{N}$ & Study & Method \\
\hline (Kasprian et al. $\overline{\text { (Ko13) }}$ & "DTI & $\overline{(20 / 20)}$ & Phite matter connectivity & Tractography \\
\hline$(\overline{J a k a b}$ et al., 2015$)$ & DTI & $(20 / 20)$ & Brain connectome organization & Tractography \\
\hline (Tarui et al., 2018) & MRI & $(7 / 17)$ & Cortical folding & $\begin{array}{l}\text { Semi-automatic segmentation, } \\
\text { sulcal pattern analysis }\end{array}$ \\
\hline (Knezović et al., 2019) & MRI & $(39 / 46)$ & Hippocampal volume & $\begin{array}{l}\text { Manual segmentation, } \\
\text { volume measurement }\end{array}$ \\
\hline
\end{tabular}

This review of the quantitative approaches to analyse volumetric fetal MRI shows that the field is mostly focused on the investigation of specific brain structures within the framework of volumetric and curvature studies. Such analyses rely heavily on brain segmentation 
and on prior hypotheses about which structures demonstrate relevant dynamics or impairments. Noteworthy exceptions include tensor-based morphometry (Rajagopalan et al., 2011, 2012; Pontabry et al., 2015; Payette et al., 2019), which considers the whole brain but only involves local shape changes, and longitudinal atlases that have so far focused on healthy brain growth, apart from (Fidon et al., 2021b). Quantitative studies of abnormal fetal brains are scarce and limited in the same way, to which CCA-specific studies are no exception: all prenatal studies have focused either on the hippocampus, cortical plate, or white matter fibers. Moreover, they often rely on small datasets. Conversely, in this paper we introduce a new approach for whole-brain, quantitative analysis of fetuses with abnormalities, and we demonstrate its effectiveness on a large dataset of fetuses with CCA.

\section{Methods}

\subsection{LDDMM framework and applications}

Our image processing and shape analysis pipeline are based on specific shape analysis tools developed in the LDDMM framework. Here, we provide a description of the theoretical setting behind these geometrical tools.

\subsubsection{LDDMMM FRAMEWORK}

The LDDMM framework (Trouvé, 1998; Christensen et al., 1996; Miller et al., 2002) is a mathematical setting to compute shape transformations. It generalizes the linearized deformation setting in order to define diffeomorphic deformations. A flow of diffeomorphisms is considered as a Riemanian Manifold of infinite dimension, and shapes are seen as objects on that manifold, transformed through deformations of the whole ambient space.

Diffeomorphisms are constructed by integrating time-dependant vector fields, considered as infinitesimal linearized deformations. Namely, to build a flow of diffeomorphisms $\phi_{t}$, one integrates the flow equation, which describes the motion of a particle $x$ along the curve $x(t)$ :

$$
\left\{\begin{array}{l}
\frac{d x(t)}{d t}=v_{t}(x(t)) \\
x(0)=x_{0} .
\end{array}\right.
$$

This model builds a flow of diffeomorphisms $\phi_{t}: x_{0} \longrightarrow x(t) \forall t \in[0,1]$. The diffeomorphism of interest $\phi_{1}$ is the end point of the path $x(t)$ :

$$
\forall x_{0} \in D, \quad \phi_{1}\left(x_{0}\right)=x(1)
$$

Here, we restrict ourselves to a finite parametrization of the velocity field $v$ (Durrleman et al., 2012). Namely, we impose that $v$ belongs to a finite dimensional subspace of a RKHS $V$, i.e. the set of square integrable functions convolved with an interpolation kernel $K_{g}$ :

$$
v_{t}(x)=\sum_{k=1}^{k_{g}} K_{g}\left(x, c_{k}(t)\right) \alpha_{k}(t)
$$


where $\left(\alpha_{k}\right)_{k}$ is a set of momentum vectors attached to $k_{g}$ control points $\left(c_{k}\right)_{k}$, and $K_{g}$ is usually a Gaussian kernel of width $\sigma_{g}$. $K_{g}$ acts as a spatial regularizer restricting the range of deformations the model is able to express.

The Riemanian manifold is provided with a right invariant metric, defined as the total kinetic energy needed between the identitity map $I_{d}$ and the diffeomorphism $\phi_{1}$, i.e.:

$$
d\left(I_{d}, \phi_{1}\right)=\int_{0}^{1}\left\|v_{t}\right\|_{V}^{2} d t
$$

To ensure that the transformation $\phi_{1}$ is diffeomorphic, regularity conditions are imposed so that the vector fields $v$ are geodesics, i.e. the shortest paths between $\phi_{0}$ and $\phi_{1}$ according to the norm $\int_{0}^{1}\left\|v_{t}\right\|_{V}^{2} d t$.

It has been shown that if an initial velocity field $v_{0}$ is written in the following manner:

$$
v_{0}(x)=\sum_{k=1}^{k_{g}}\left(x, c_{k}(0)\right) \alpha_{k}(0),
$$

then the vector fields $v_{t}$ along geodesic paths of direction $v_{0}$ remain defined as a linear combination of RKHS basis elements (Miller et al., 2006):

$$
v_{t}(x)=\sum_{k}\left(x, c_{k}(t)\right) \alpha_{k}(t)
$$

Further, the kinetic energy along geodesic paths is preserved over time, i.e. $\forall t \in$ $[0,1],\left\|v_{t}\right\|_{V}=\left\|v_{0}\right\|_{V}$. Moreover, the evolution of the control point positions $\left(c_{k}(t)\right)_{k}$ and momentum vectors $\left(\alpha_{k}(t)\right)_{k}$ satisfy Hamiltonian equations that describe the movement of a set of particles, with $K_{g}$ modeling the forces of repulsion between particles.

It follows that the vector fields along geodesics paths are fully parametrized by the initial velocity field $v_{0}$. This has two main consequences:

(1) $v_{0}$ is the tangent space representation of the diffeomorphism $\phi_{1}$ at the identity map $I_{d}$, which enables one to define tangent-space statistics of the diffeomorphism $\phi_{1}$ and to characterize $\phi_{1}$ with standard statistical tools such as Principal Component Analysis.

(2) to estimate $\phi_{1}$, one only needs to estimate the system's initial conditions $\alpha_{0}=\left(\alpha_{k}(0)\right)$ and $c_{0}=\left(c_{k}(0)\right)$. In other words, to compute shape changes, one solves a geodesic shooting problem: given a set of initial momentum vectors and control points characterizing a flow of diffeomorphisms, we compute the trajectory of a given shape. The end point of this trajectory, i.e. the final deformed image, is then compared to the target shape, and the initial conditions can be modified accordingly.

In this framework, optimization is performed by minimizing a cost function whose formulation depends on the task at hand (e.g. registration, geodesic regression, atlas estimation). It is typically composed of a data fidelity term, e.g. the Euclidean $l^{2}$ distance between the images we mean to map onto each other, plus a term penalizing the kinetic energy of the deformation. Optimization is performed through gradient descent. Computation of the gradients with respect to the parameters is facilitated by the fact that geodesic vector fields are always expressed as a linear combination of momentum vectors. 
The geometrical tools provided by the LDDMM framework are available in the opensource software Deformetrica (Bône et al., 2018). In the following, we will briefly detail the ones used in this work, namely registration, geodesic regression and parallel transport.

\subsubsection{REgistration}

Registration seeks the transformation $\phi_{1}$ that best warps a source image $I_{1}$ onto a target image $I_{2}$. The control points $c_{0}^{1}$ and momenta $\alpha_{0}^{1}$ that define $\phi_{1}$ are optimized by minimizing a cost function $E$ :

$$
E\left(c_{0}, \alpha_{0}\right)=\frac{\left\|I_{2}-I_{1} \circ \phi_{1}^{-1}\right\|^{2}}{\sigma^{2}}+\int_{t=0}^{1}\left\|v_{t}\right\|_{V}^{2},
$$

where $\sigma$ controls the trade-off between the two terms. The first term is the sum of squared differences between the deformed source image and the target image. The second term penalizes the kinetic energy of the transformation, ensuring that only geodesic vector fields are considered as potential solutions.

\subsubsection{Geodesic Regression}

Geodesic regression can be seen as the generalization of linear regression to shapes. Given a set of $N$ images $\left(I_{i}\right)_{1 \leq i \leq N}$ observed at times $\left(t_{i}\right)_{1 \leq i \leq N}$, we seek the geodesic trajectory $\gamma_{t}$, defined by control points $c_{0}$ and momentum vectors $\alpha_{0}$ that best fit the images.

The cost function is defined as follows:

$$
E\left(c_{0}, \alpha_{0}\right)=\sum_{i=1}^{N}\left(\frac{\left\|I_{i}-I_{1} \circ \phi_{t_{i}}^{-1}\right\|^{2}}{\sigma^{2}}\right)+\int_{t=0}^{1}\left\|v_{t}\right\|_{V}^{2},
$$

We refer to $I_{1}$ as the template image: it is the point from which the trajectory is computed. Note that $I_{1}$ is user-defined and can be any of the images in the set $\left(I_{i}\right)_{1 \leq i \leq N}$.

\subsubsection{PARALlEL TRANSPORT}

Parallel transport is a differential geometry notion that considers two known transformations $\gamma_{t}$ and $\phi_{2}$ defined by the sets of parameters $\left(c_{0}^{1}, \alpha_{0}^{1}\right)$ and $\left(c_{0}^{2}, \alpha_{0}^{2}\right)$, respectively. Typically, $\gamma_{t}$ describes the known evolution of a reference shape, and $\phi_{2}$ describes the transformation that maps the reference subject at a given time to a new subject. Parallel transport enables one to transport the diffeomorphism $\phi_{2}$ at any time point along the reference trajectory. We denote by $T P^{\gamma}\left(\alpha_{0}^{2}\right)\left(t_{i}\right)$ the Parallel Transport of momentum vectors $\alpha_{0}^{2}$ along the trajectory $\gamma_{t}$ at time $t_{i}$. The geodesic shooting of the transported momenta can be used to predict the shape of a new subject at any time $t_{i}$, defining a trajectory that is parallel to the reference one. For further details about the computation of Parallel Transport, the reader is referred to (Louis et al., 2017).

\subsection{Data acquisition}

Data. Data consist of retrospectively selected fetal MRIs acquired between 2006 and 2019 at hospital Armand Trousseau, in Paris, France. The database contains 38 healthy fetuses scanned between 26 and $37 \mathrm{GW}$ (mean $=32.4 \pm 1.69$ ) and 73 fetuses diagnosed with CCA 


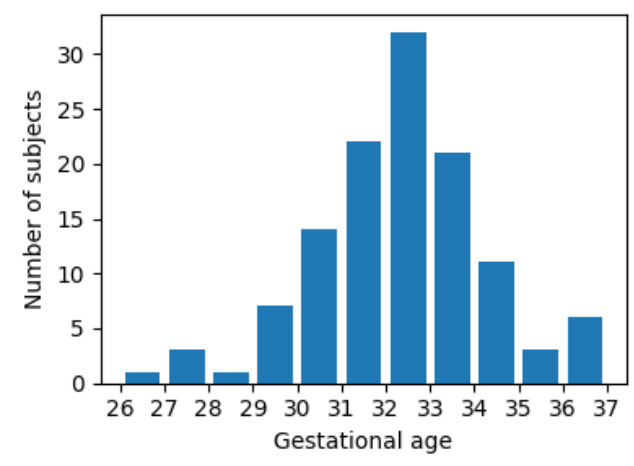

Figure 1: Histogram of the subjects gestational ages at the date of MRI

scanned between 25 and $37 \mathrm{GW}$ (mean $=31.63 \pm 2.09$ ). A histogram of the subjects gestational ages is presented in Fig. 1. Healthy fetuses had normal central nervous system findings at MRI examination. For the group with CCA, MRI acquisitions were performed as part as clinical routine after findings of abnormality of the corpus callosum identified at second (between 22 and $24 \mathrm{GW}$ ) or third trimester (between 31 and $33 \mathrm{GW}$ ) screening ultrasound examination. The routine ultrasound was followed by an expert ultrasound assessment including neurosonography to investigate other associated fetal anomalies before MRI scanning. Patients had a consultation with a fetal medicine specialist, a pediatric neurologist and a clinical geneticist in the referral prenatal center and were offered amniocentesis for fetal karyotyping and chromosomal microarray. Corpus callosum anomalies were defined as: (1) complete CCA, namely the complete absence of the corpus callosum and (2) partial CCA, i.e. the absence of one or more of the five segments of the corpus callosum resulting in an abnormally shaped corpus callosum. Inclusion criteria were as follows: fetuses affected by isolated or associated partial or complete corpus callosum abnormalities. Fetuses with short corpus callosum, defined as a complete corpus callosum with an anteroposterior diameter below the third percentile, were not included in this study. In the group with abnormal corpus callosum, 51 fetuses have partial CCA and 22 complete CCA.

Image acquisition. Fetal brain MRI was performed using repeated T2 half-Fourier Single Shot Fast Spin Echo (SSFSE), or Single-Shot half-Fourier Turbo Spin Echo (SshTSE). MRIs were acquired on a 1.5 T MRI system Achieva Philips (Best, the Netherlands) before 2016 and Optima MR450w General Electric (Waukesha, WI, USA) after 2016. Maternal sedation was systematically offered to reduce fetal motion artefacts. Scan acquisitions were performed in the three orthogonal planes. Scanning parameters were as follows: field of view: $256 \times 256$ or 512x512 mm; echo time: 150-200 ms; repetition time: 3500-4000 ms; slice thickness: $4 \mathrm{~mm}$; flip angle: $90^{\circ}$; acquisition matrix: 320x320. To ensure proper volumetric reconstruction, only images with at least 3 stacks in the 3 different orientations are included in the analysis.

\subsection{Image processing pipeline}

In order to perform quantitative analyses, brain volumes are reconstructed from the 2D fetal images, and the volumetric image is processed further to enable inter-subjects comparisons. 


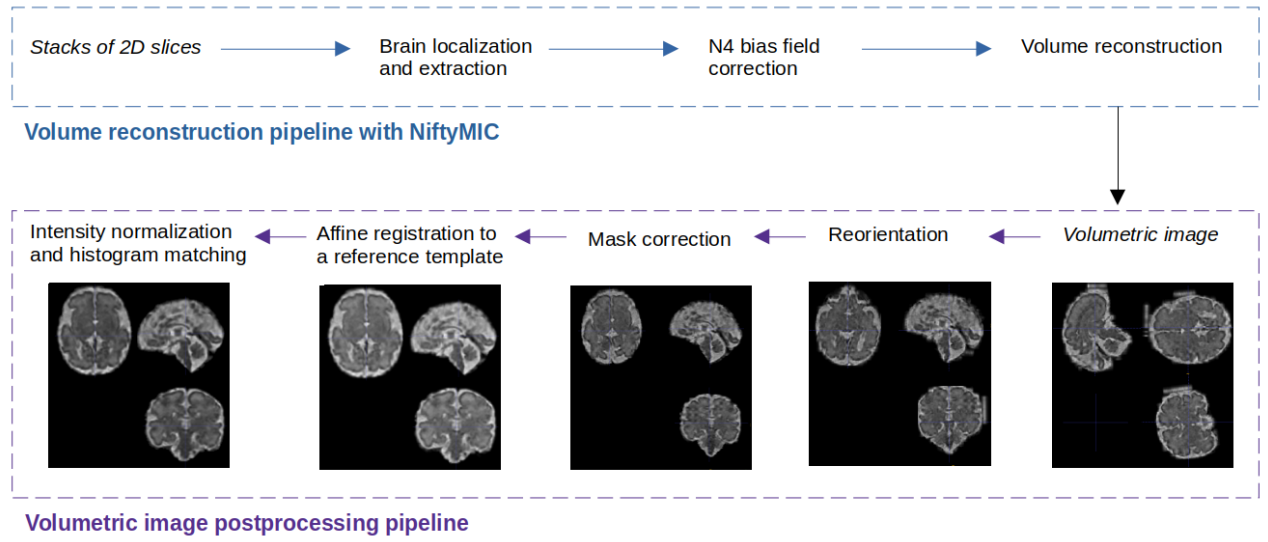

Figure 2: Overview of the fetal MRI preprocessing steps

The steps of these volume reconstruction and image processing pipelines are summarized in Fig. 2 and detailed below.

\subsubsection{Semi-Automatic volume Reconstruction Pipeline}

Brain extraction and volume reconstruction. Isotropic high resolution 3D volume reconstruction of fetal brains is performed using the open-source state-of-the-art NiftyMIC software (Ebner et al., 2020) that takes as input stacks of low resolution 2D slices. First, brain extraction is performed in each $2 \mathrm{D}$ stack with a coarse-to-fine approach that localizes and then extracts the fetal brain using two CNNs. Intensity non-uniformity is corrected using N4 bias field correction. Finally, high resolution reconstruction is performed by iterating between SVR registration for motion correction and SRR with robust rejection of slices that are misregistered or corrupted by artifacts.

Reconstruction performance on our dataset. Our dataset is composed of MRIs acquired during clinical routine, destined for visual examination of $2 \mathrm{D}$ slices. Although the reconstruction algorithm of (Ebner et al., 2020) was successfully tested on healthy fetuses and fetuses with spina bifida, it yields many erroneous volumetric images on our dataset: $74 \%$ of brains with CCA and $40 \%$ of healthy brains have erroneous reconstructed images, as illustrated on Fig. 3. Most of the time, this is caused by incorrect delineation of the brain, which has a high rate of false positive voxels (see Fig. 4, Subjects 2 and 3 for examples).

\section{Correction of erroneous brain extraction.}

Instead of correcting manually the volumetric brain masks, which is very time consuming, we design a semi-automatic volume reconstruction pipeline, which is described in Fig. 3. In cases where brain extraction with NiftyMIC is erroneous, we reiterate the extraction step using the U-net CNN from (Salehi et al., 2018). U-net is affected by the same defect as NiftyMIC as it yields a high proportion of false positive voxels, and was deemed less efficient than NiftyMIC in previous experiments (Ebner et al., 2020). Thus, our goal is not to extract a more relevant brain mask with the U-net algorithm, but to fuse the extraction results from U-net and NiftyMIC. Fusion is carried out in the following manner: brain extraction is performed with NiftyMIC and U-net, and two brain volumes are reconstructed; we then 


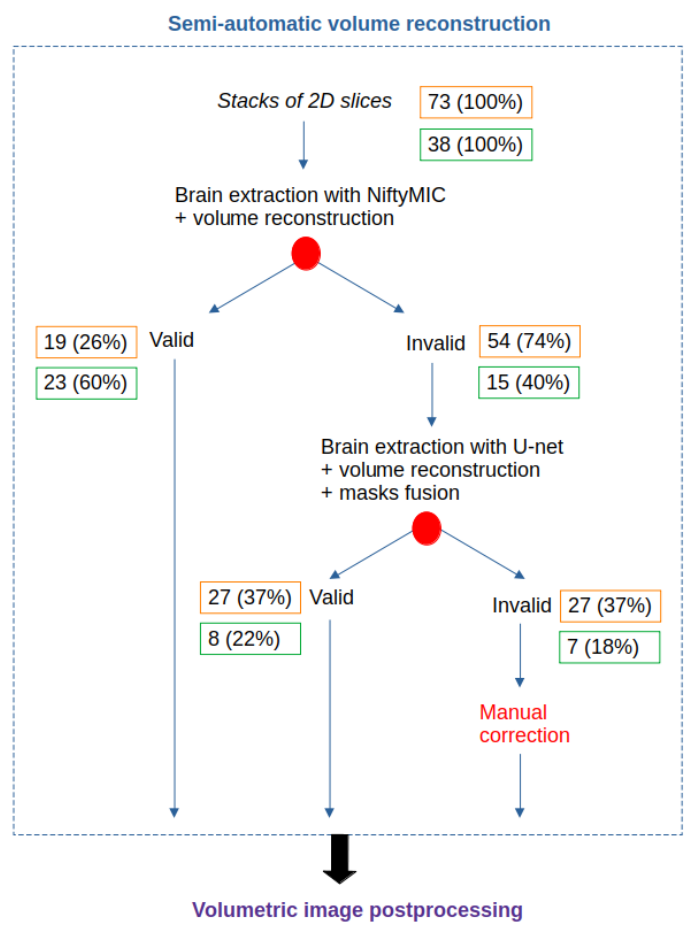

Figure 3: Steps of the semi-automatic volume reconstruction pipeline. Numbers in orange and green rectangles denote the number and proportion of subjects that went through each step of the pipeline in the group with CCA and the control group, respectively. Red color denotes the user intervention: red circles imply a visual evaluation of the quality of brain extraction and volume reconstruction, followed by a decision either to keep the reconstructed image, or to try another reconstruction method.

extract the two volumetric masks yielded by both reconstructions; the intersection of the masks is computed and used to re-mask the two volumetric images. Visual examinations of the re-masked images are performed and the reconstruction with highest quality is selected. This mask fusion procedure is illustrated the second column of Fig. 4.

In $37 \%$ of fetuses with CCA and $18 \%$ of healthy fetuses, the mask fusion yields poor results, characterized by the incomplete elimination of false positive voxels. In such cases, manual refinement of the volumetric masks are performed using ITK-SNAP, Version 3.6 (Yushkevich et al., 2016), in approximately 10 minutes per subject. Note that the mask fusion step renders the manual correction less time-consuming as it eliminates large amounts of false positive voxels. The third column of Fig. 4 shows an example of a fetal brain for which manual correction was performed. In this worst-case scenario, the semi-automatic reconstruction pipeline takes approximately one hour per subject, compared with 20 minutes in the optimal scenario.

\subsubsection{Volumetric image Postprocessing PiPeline}

We now introduce our fully automated image processing pipeline, that aims at enabling comparisons between subjects. This pipeline is summarized in the bottom row of Fig. 2 and illustrated in the three bottom rows of Fig. 4.

Reorientation. As the fetus orientation is unknown during image acquisition, it is necessary to identify the coronal, sagittal and axial planes of the reconstructed image and rotate them if necessary. First, the inferior-superior, antero-posterior and right-left axis are automatically identified based on length and symmetry measurements: the size of the brain is computed along each of the three axes, and the antero-posterior axis is identified 
as the axis with the highest length measure. Then, symmetry indices are computed along the two remaining axes, and the right-left axis is identified as the axis with the highest symmetry index. Finally, we must ensure that each of the axes is correctly oriented. To reorient the antero-posterior and inferior-superior axes, we extract the $2 \mathrm{D}$ median sagittal plane. Areas of the putative anterior half and posterior half of the brain are compared, and the antero-posterior axis is flipped if the surface of the putative anterior half is larger than that of the putative posterior half. Similar area measurements on the putative superior and inferior halves of the brain are performed in order to reorient the inferior-superior axis.

Mask correction. The subject brains are aligned and cropped to a size of 105x100x120 voxels. To correct small errors (i.e. false positive voxels) during the brain extraction step, a correct brain mask is extracted from the reference brain of (Gholipour et al., 2017), rigidly registered to each erroneous fetal brain mask using Deformetrica, Version 4.3.0 (Bône et al., 2018), and used to re-mask the fetal brain. Note that this automatic mask correction step can only be applied to mildly erroneous brain masks (see Subject 1 in Fig. 4) as the overall shape of the brain has to resemble that of the template brain. Brain masks that are already correct, such as that of Subject 3 in Fig. 4, go through this step without being affected.

Affine registration to a reference template. To enable inter-subjects comparisons and eliminate position and size differences, fetal brains are spatially normalized by performing affine registration to a common anatomical space, namely the template brain at $31 \mathrm{GW}$. Finally, intensity normalization and histogram matching to the template are performed.

\subsection{Shape analysis}

Shape analysis pipeline. Registering a reference average brain, called template, to healthy or pathological brains, yields transformations that encode subject-specific anatomical deviations from normality. As brains undergo important structural changes during gestation, we compare each fetal brain to a healthy template of the same age using registration. To enable inter-subjects comparisons, deformations are transported to a common space using parallel transport. PCA is applied to the transported subject deformations to reduce dimension and extract relevant features. Finally, these features are fed to a SVM to perform patient classification. The steps of our shape analysis pipeline are summarized in Fig. 5 and detailed below. Shape transformations, namely geodesic regression, registration and parallel transport, are computed using the open-source software Deformetrica, Version 4.3.0 (Bône et al., 2018). Movies of the different steps of the shape analysis pipeline are available at the first author's webpage ${ }^{1}$

Geodesic regression of template brains. To take into account the anatomical changes that occur during gestation, each fetal brain is compared to an age-matched healthy brain. We use as reference a spatiotemporal atlas defined at each week of gestation, constructed from 81 healthy fetuses scanned between 19 and 39 GW (Gholipour et al., 2017). We extract the 13 template brains between 26 to $38 \mathrm{GW}$ and spatially normalize them to the space of the template at age 31. From this discrete set of templates, we construct a continuous trajectory of normal brain changes from 26 to $38 \mathrm{GW}$ by performing geodesic regression, which is described in Section 3.1.3. This trajectory $\gamma(t)$ (red curve in Fig. 5) is described by a pair of vectors, namely the control points $c_{0}$ and momenta $\alpha_{0}$ defined at

1. https://fleurgaudfernau.github.io/Shape_analysis/ 


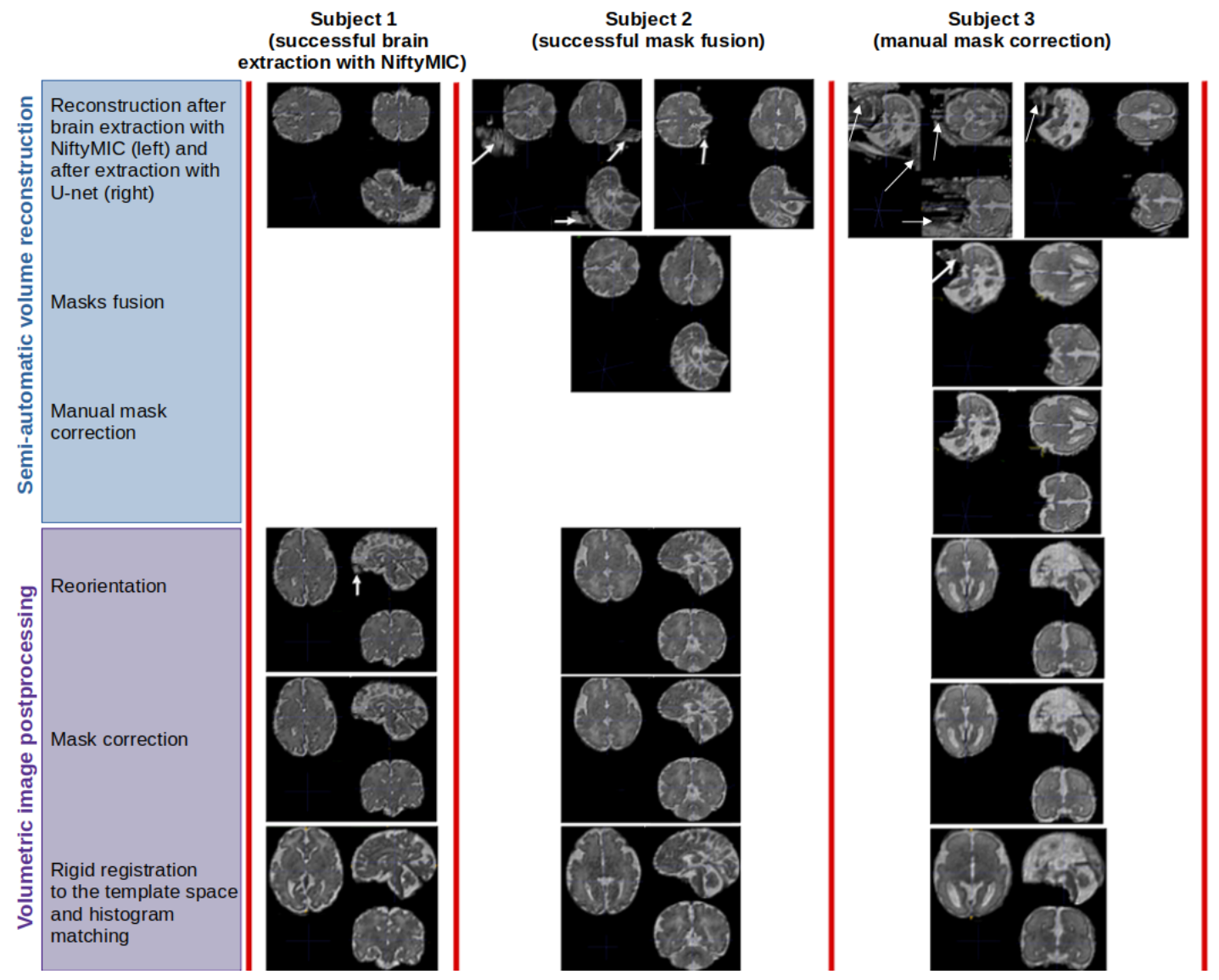

Figure 4: Illustration of the fetal MRI processing pipeline on three examples. Top three rows: semi-automatic volume reconstruction pipeline. Three bottom rows: volumetric image postprocessing pipeline. First column: reconstruction of Subject 1 after brain extraction with NiftyMIC yields a good quality image and no mask correction is needed. Second column: volumetric images of Subject 2 obtained after brain extraction with NiftyMIC (left) and U-net (right). The intersection of the two erroneous masks is computed, yielding a correct volumetric mask. Third column: volumetric images of Subject 3 obtained after brain extraction with NiftyMIC (left) and U-net (right). Fusion of the two erroneous masks does not eliminate all false positive voxels, hence manual correction is performed. White arrows indicate groups of voxels erroneously classified as fetal brain). 


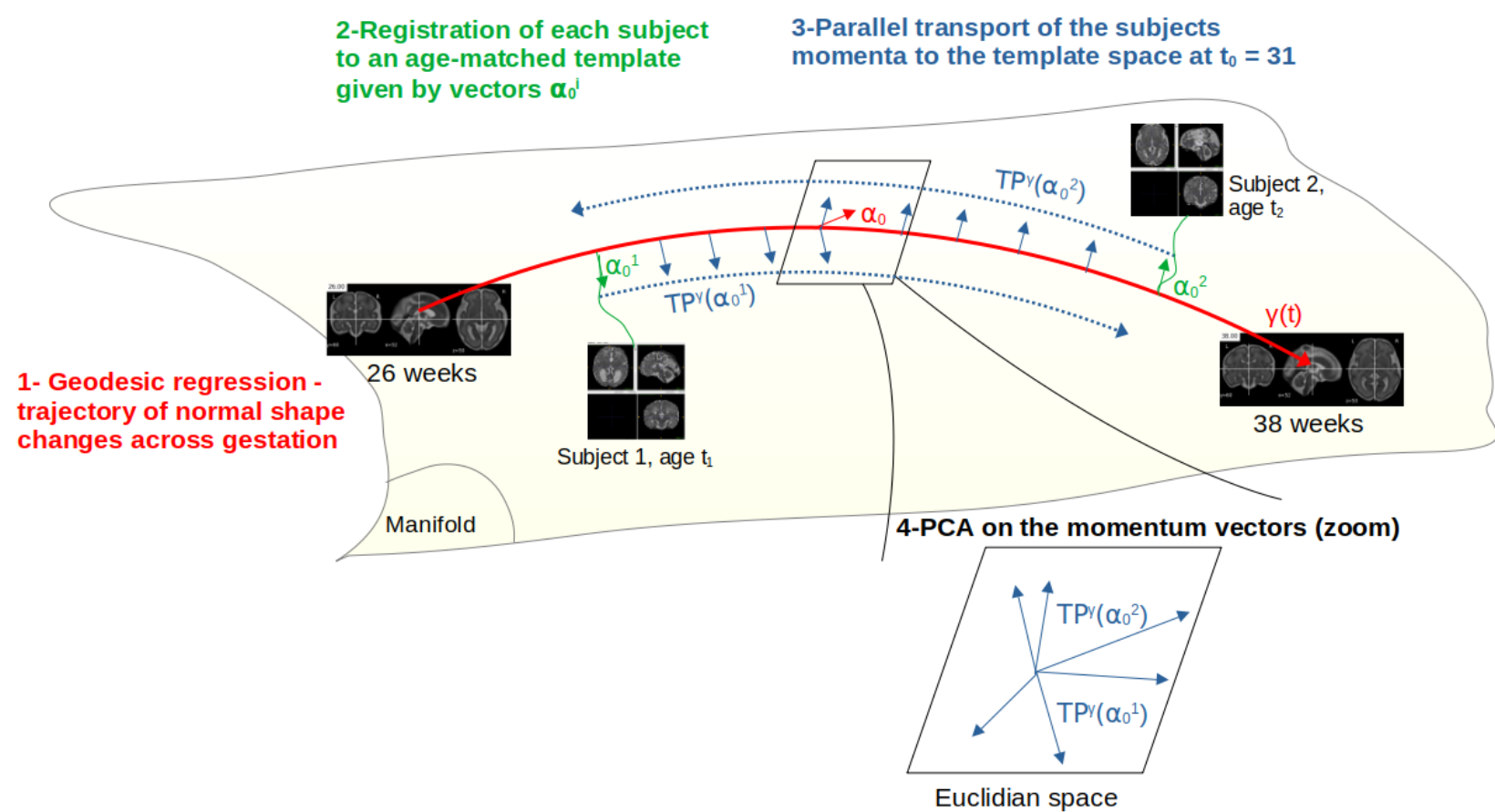

Figure 5: Shape analysis pipeline

time $t_{0}=31 \mathrm{GW}$. The point from which the geodesic is computed, i.e. the template brain at age 31 , is chosen arbitrarily and will be referred to as $T_{r e f}$ in the following.

Registration to an age-matched template. For each subject i, the age-matched template is extracted from the geodesic trajectory, and registered (see Section 3.1.2) to the subject's brain using geodesic shooting. Given an initial set of controls points $c_{0}^{i}$ and momenta $\alpha_{0}^{i}$, geodesic shooting computes the trajectory of a shape under the flow of diffeomorphisms defined by $c_{0}^{i}$ and $\alpha_{0}^{i}$ (green paths). By comparing the deformed template image and the subject image, registration optimizes the $c_{0}^{i}, \alpha_{0}^{i}$ that best warp the template image to match the subject image. $\mathrm{P}=10,000$ control points are used for the registration, which corresponds to a 5 voxel spacing.

Parallel transport. The diffeomorphism computed by registration encodes, for each subject, the difference between its anatomy and that of an age-matched healthy brain template. However, to enable comparisons between subjects, transformations need to exist in the same space. The momenta parametrizing each deformation are parallel transported to the tangent space of $T_{r e f}$. In brief, parallel transport (see Section 3.1.4) translates the deformation towards subject i, defined by $c_{0}^{i}$ and $\alpha_{0}^{i}$, at any time point along the trajectory $\gamma(t)$ (blue arrows). It adjusts for anatomical differences related to gestational age while preserving components of the transformation non-related to age. In other words, parallel transport provides a way of artificially transporting the subjects anatomies to the same gestational stage.

PCA. Given the high dimension of the transformations ( $\mathrm{P}=10,000$ control points) and the low sample size $(\mathrm{N}=111)$, the momenta cannot be used as features to perform prediction. 


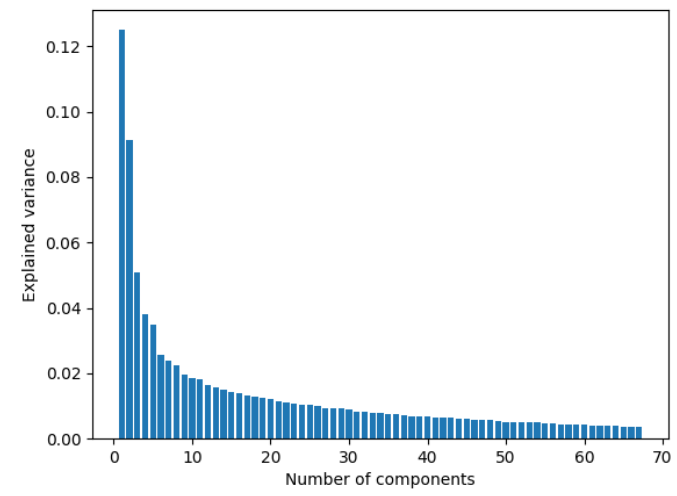

Figure 6: Explained variance of each PCA component

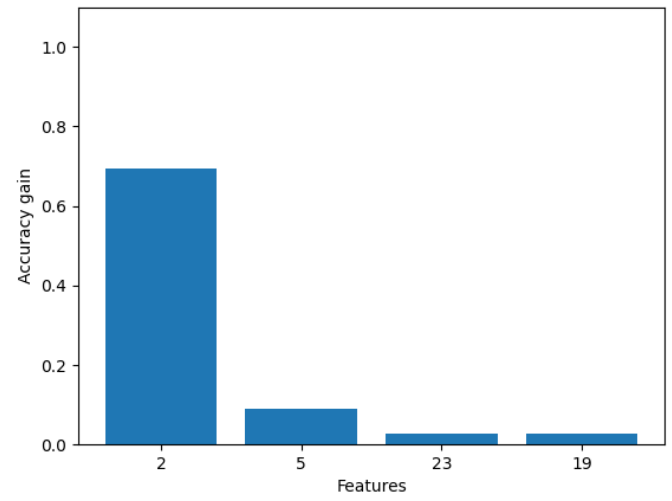

Figure 7: Accuracy gain for each feature added to the model

To reduce feature space and extract interpretable deformation modes, PCA is applied to the subjects deformation fields.

We denote by $\beta_{i}$ the $3 P$ transported momentum vector of subject $i$ and we introduce the $N$ by $3 P$ matrix of transported momenta: $X=\left(\beta_{1}, \ldots, \beta_{N}\right)^{T}$. The empirical mean of the transported momenta is given by: $\bar{\beta}=\sum_{i=1}^{N} \frac{\beta_{i}}{N}$. We introduce the mean-centered matrix of transported momenta $Z$, defined as: $Z=\beta_{i}-\bar{\beta}$.

The $3 P$ by $3 P$ empirical covariance matrix is given by $\Sigma=Z^{T} Z$. Eigendecomposition of $\Sigma$ is performed in the form of $\Sigma=U \Lambda U^{-1}$, in which $U$ is a matrix of size $3 P$ by $N$, whose columns $\left(U_{1}, \ldots, U_{n}\right)$ are the eigenvectors of $\Sigma$, and $\Lambda$ a diagonal matrix of size $N \times N$, whose diagonal elements $\left(\lambda_{1}, \ldots, \lambda_{n}\right)$ are the eigenvalues of $\Sigma$. Each eigenvector $U_{k}$ is associated to an eigenvalue $\lambda_{k}$, representing the amount of variability that is explained by $U_{k}$. We extract the first 67 components that characterize $90 \%$ of the sample shape variability (see Fig. 6).

Deformation modes. Being a linear combination of momentum vectors, each eigenvector can generate a diffeomorphism, called deformation mode, which represents how the template brain anatomy varies within the population.

The $i^{t h}$ mode is given by: $m_{i}=\bar{X}+c \sigma_{i} U_{i}$, with $c \in[-4,-2,0,2,4], \sigma_{i}=\sqrt{\lambda_{i}}$, and $U_{i}$ the $i^{\text {th }}$ eigenvector. In order to visualize the deformation mode $m_{i}$, we apply the generated diffeomorphism to the template brain $T_{\text {ref }}$.

In T2 fetal MRI, thinness and hypointensity of the corpus callosum make it difficult to discern. Geodesic shooting is performed on the template segmentation image as provided by (Gholipour et al., 2017) to make corpus callosum deformations discernible.

Projection of the momenta of subject $j$ on deformation $i$ is computed as follows: $P_{\beta_{j}}=\beta_{j}^{T} U_{i}$. $P_{\beta_{j}}$ can be seen as a score quantifying how much $\beta_{j}$ is represented by the $i^{\text {th }}$ deformation mode.

Classification. To assess whether or not the deformation modes can discriminate between controls and fetuses with CCA, we perform classification with a SVM equipped with a RBF kernel, that receives as input the subjects scores on the deformation modes. SVM parameters (width of the gaussian kernel and penalty) are tuned using grid-search. The 
dataset is randomly split into a training ( $70 \%$ of the data) and a test set (30\% of the data) to perform 5-fold cross validation. While modes with the highest eigenvalues are those that explain best the anatomical variability of the data, they do not only encode shape variations related to CCA, but also components of rigid registration correction and inter-subject variability. To extract deformation modes specific to CCA, we perform forward feature selection: starting from an initial model with no input features, we train the model with each of the 67 principal deformations independently and keep the one that best enhances the model accuracy. This process is repeated iteratively until the addition of a new deformation does not augment the accuracy. This leads to the selection of 4 deformation modes as indicated in Fig. 7 .

\section{Results}

The final classification model reaches a 90\% ( $\pm 7 \%$ ) accuracy. Interestingly, feature selection did not retain the first component of PCA, which accounts for $12 \%$ of the sample shape variability. Visual inspection of the related deformation mode (presented in Appendix 6) indicates it corrects for brain misalignment and characterizes subjects with large ventricles.

We present in Fig. 8 the second component, which drives to most of the model accuracy (see Fig. 7). The segmentation image of the template brain is transformed by the second mode of deformation in directions $-4 \sigma$ and $-2 \sigma$, on which healthy subjects generally score higher, and in directions $+2 \sigma$ and $+4 \sigma$, on which subjects with CCA generally score higher. Complete movies of these deformation modes are available at the first author's webpage ${ }^{2}$. Of note, the score distributions of subjects with CCA is more spread out than that of control subjects. While healthy fetuses are mostly characterized by negative scores, fetuses with CCA reach a wider range of values.

The direction of deformation that mostly characterizes subjects with CCA reveals a thinning and a shortening of corpus callosum (C) on sagittal view. It is folded into a $\mathrm{V}$ like shape, with a stronger distortion towards its posterior part. Volume of the cingulate gyrus $(G)$ is also reduced. Lateral ventricles $(V)$ are widely spaced and parallel, with prominent occipital horns and atrium, corresponding to colpocephaly. Dilation is slightly stronger in the right ventricle. Volume of the occipital cortical and subcortical region $(\mathrm{O})$ is reduced, especially in the right hemisphere. Hippocampi $(\mathrm{H})$ appear thinner and verticalized. The superior temporal sulci $(\mathrm{S})$ seems less pronounced. On coronal view, thalami $(\mathrm{T})$ are parallelized and displaced away from the interhemispheric fissure. Shape of the brainstem (B) is abnormal on sagittal view, with prominent pons and midbrain.

\section{Discussion}

In this work, we addressed the challenge of exploring quantitatively alterations in abnormal fetal brains. We developed a semi-automatic volume reconstruction pipeline, together with a shape analysis pipeline that are adapted to the specificities of clinical fetal MRI.gyrification Geometrical models based on diffeomorphisms, that were originally designed for postnatal imaging, enabled us to compare fetuses of different ages and investigate brain alterations globally, without requiring any prior assumption. Such models are adapted to the scarcity

2. https://fleurgaudfernau.github.io/Shape_analysis/ 

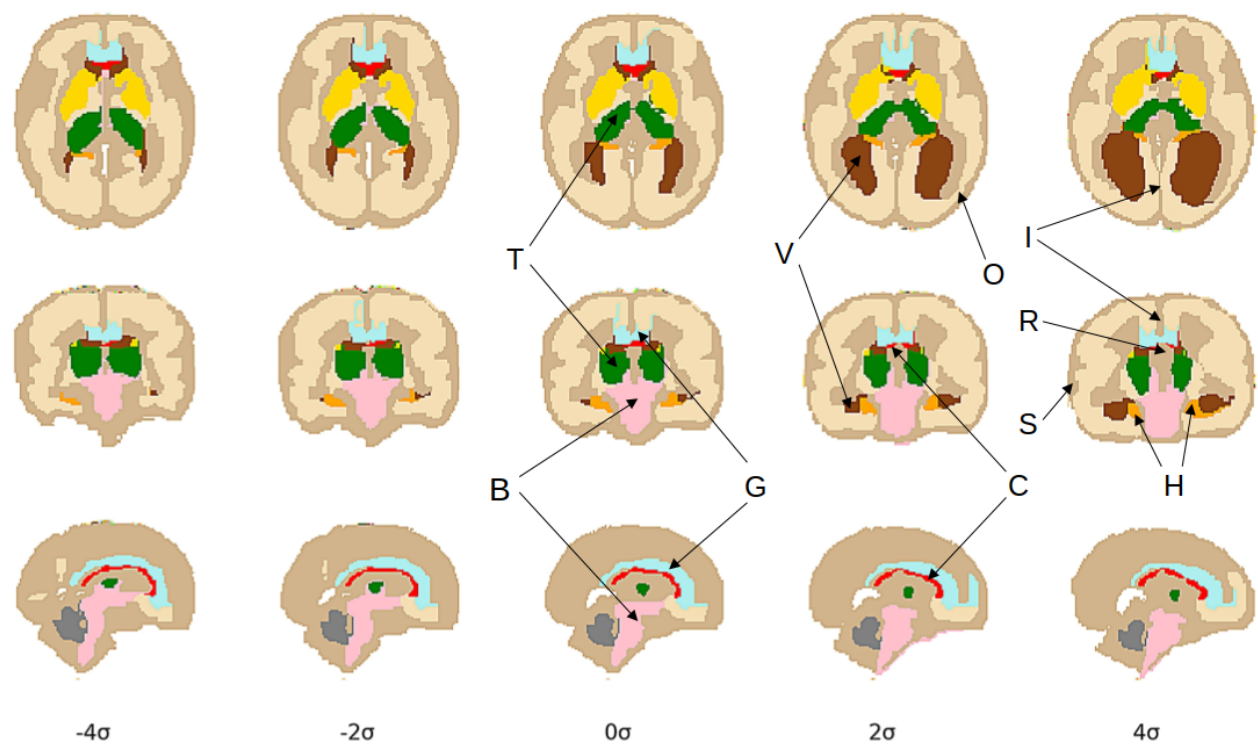

$-4 \sigma$

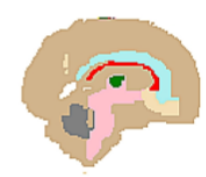

$-2 \sigma$

$0 \sigma$

$2 \sigma$

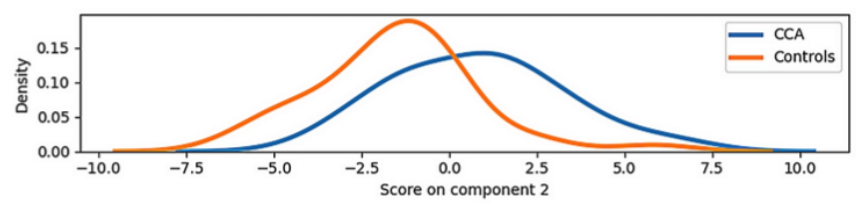

$4 \sigma$

Figure 8: Second mode of deformation applied to the segmentation of the template at age 31 GW. Top three rows: axial, coronal and sagittal views. Leftmost columns: geodesic shooting of the template by the second mode of deformation at $-4 \sigma$ and $-2 \sigma$ (characterizing healthy subjects). Central column: template brain. Rightmost columns: geodesic shooting of the template brain by the second mode of deformation at $+2 \sigma$ and $+4 \sigma$ (characterizing subjects with CCA). Bottom row: distribution of the subjects scores on component 2. B: brainstem. C: corpus callosum. G: cingulate gyrus. H: hippocampi. I: interhemispheric fissure. O: occipital cortex. R: roof of the third ventricle. S: superior temporal sulcus. T: thalami. V: lateral ventricles.

of medical data and to the need for interpretable results. This preliminary work opens new perspectives for the quantitative analysis of fetal brains with developmental alterations.

\subsection{Fetal MRI preprocessing}

In this paper, particular attention was given to data preprocessing. As our analysis draws on whole-brain shape comparisons, the accuracy of brain extraction and alignment can impact the results and is thus of prime importance.

This analysis exploited retrospectively selected fetal MRIs acquired during clinical routine. Although we used a state-of-the-art processing pipeline (Ebner et al., 2020) for brain extraction and volume reconstruction, the brain extraction algorithm showed poor results on our dataset. The reconstruction task was less challenged by healthy fetal brains, with 
$60 \%$ of correct reconstruction following brain extraction by NiftyMIC, and only $18 \%$ of manual correction required. This suggests that brain extraction algorithms are less robust to developmental defects. However, it should also be noted that healthy fetuses in our dataset were imaged more recently (i.e. after 2015) than fetuses with CCA, which likely led to higher image quality.

Due to the significant number of images in our dataset, we chose to correct erroneous brain delineation in an automatic manner whenever possible, instead of the commonly employed manual correction. We introduced a novel semi-automatic reconstruction pipeline that takes advantage of two brain extraction algorithms (Ebner et al., 2020; Salehi et al., 2018) and merge their volumetric brain masks. This mask fusion was applied to 69 brains masks, among which 34 had to be further refined. Our semi-automatic procedure, though time-consuming, is more efficient than performing directly manual correction: in most cases, only small corrections were required, which took in average 10 minutes, whereas fully manual volumetric brain extraction has been reported to take 2 to 5 hours (Hu et al., 2011).

With this pipeline, we illustrate that the existing preprocessing methods for fetal brain MRI still lack maturity and robustness. While current methods are efficient on high quality research data, they may show poor results on routinely acquired images originating from impaired subjects. Even in recent years, some quantitative studies have favored manual or semi-automatic approaches during image processing, whether it be for brain extraction (Payette et al., 2019), alignment (Yun et al., 2018) or reorientation (Kyriakopoulou et al., 2017). Further evaluation of our data preprocessing pipeline will be done to achieve reproducible quantitative studies of fetal brains.

\subsection{Deformation models applied to fetal brains}

In this work, we applied for the first time deformation models based on diffeomorphisms to abnormal fetal brains. One of the main advantages of this approach is that it provides a novel and practical way of dealing with the gestational age heterogeneity in datasets of fetal images by transporting subjects-specific deformations to a common space.

Another benefit linked to deformation models is that it enabled us to target the whole brain. Hence, our method does not require tedious manual segmentations nor automatic ones, which are less reliable on brains with malformations (Fidon et al., 2021a). This is in contrast to previous studies on brains with CCA (Bénézit et al., 2015; Warren et al., 2010; Knezović et al., 2019; Tarui et al., 2018; Nakata et al., 2009; Schwartz et al., 2021), which often had fewer data and focused on specific brain areas or structures. It is also important to note that unlike most papers, our analysis is not restricted to the study of tissue volume changes. While our approach is related to tensor-based morphometry (Rajagopalan et al., 2011, 2012), the latter only reflects local volume changes, while our pipeline also includes global transformations, therefore our methodology provides richer and more complex information about anatomical alterations.

The geometrical tools we employed come with several limitations. As the registration was computed in the space of the healthy template brain using topology-preserving deformations, structures specific to brains with CCA such as Probst's bundles could not be studied. Moreover, parallel transport assumes that the speed of growth of impaired fetal brains is similar to that of healthy brains, which is is contradiction with reported growth delays for 
fetal brains with CCA (Tarui et al., 2018). To strengthen the methodology, spatiotemporal models (Debavelaere et al., 2020) will be adapted to take into account subject-specific growth rates. Furthermore, the small spacing between control points yielded unregular deformations, that can be anatomically inaccurate. Methods based on multiscale vector flows, which have been shown to produce more realistic deformations (Debroux et al., 2021), are currently under consideration.

\subsection{Anatomical variability of fetal brains with CCA}

Our method extracted a mode of deformation that depicts the anatomical variability related to the health status of the fetuses in our dataset.

First, the distribution of the scores of subjects with CCA on the second component of PCA was more widespread than that of control subjects. This might reflect the greater anatomical variability of abnormal fetuses compared to healthy ones.

The second component generated global deformations that correlate together, as they belong to the same deformation mode. These alterations revealed well-known defects of brains with CCA. As expected, the corpus callosum had abnormal shape and size. It was especially distorted in its posterior segment, the splenium, which is usually the missing part in partial CCA (Raybaud, 2010). The cingulate gyrus, commonly absent in CCA (Bénézit et al., 2015), was also reduced. As our dataset comprised fetuses with complete and partial CCA, it cannot be known whether these patterns reflect a reduction or an absence of both structures. CCA is often accompanied by the development of a pair of aberrant callosal fibers, called Probst bundles, that run parallel to the midline, and a rearrangement of the midline cerebral structures (Leombroni et al., 2018). The most common alterations include colpocephaly (Leombroni et al., 2018; Bénézit et al., 2015), which was clearly visible in the second deformation mode. Ventricles dilation and volume reduction of the occipital cortical and subcortical brain matter were uneven across hemispheres, which may reflect a tendency for abnormal brain asymmetry (Glatter et al., 2021; Schwartz et al., 2021). The observed volume reduction of the occipital region coincides with findings of decreased thickness of the cerebral wall in the lateral occipital region (Schwartz et al., 2021). Consistent with findings of abnormal shape and rotation of the hippocampi in fetuses with CCA (Glatter et al., 2021; Knezović et al., 2019), we observed verticalized hippocampi, probably because of the extension of the temporal ventricular horns into the parahippocampal gyri. Both observations might be related to reduced volume of the ventral cingulum bundle, the fibers of which normally have an initial course below the body of the corpus callosum and then course within the parahippocampal gyrus in the inferior and medial temporal lobe (Nakata et al., 2009). We also observed underdeveloped superior temporal sulcus, which might be related to delayed sulcation (Warren et al., 2010) or altered cortical folding (Tarui et al., 2018). Verticalization and displacement of the thalami, which are not reported in the literature, probably result from the widening of the interhemispheric fissure. It has been suggested that in CCA other interhemispheric connections, such as indirect thalamic nuclei connections, supply the absence of callosal fibers (Bénézit et al., 2015). Understanding whether the displacement of the thalami is a marker of the absence or presence of such indirect connections and related to neurodevelopmental outcome could help understand the differences in outcome of patients with apparently isolated CCA. Surprinsingly, we observed 
a strong deformation of the brainstem, which is not a typical feature of CCA. This result likely originates from inaccurate segmentation of the brainstem during image processing, which tended to exclude the medulla.

Together, our findings draw a typical profile of brains with CCA, which is in agreement with the results of more local methods, validating our approach. Our method could help understand the mechanisms of the rearrangements linked to CCA, and, above all, identify the anatomical defects related to poor clinical outcome in isolated CCA.

Of note, the number of subjects in the control group was lower than in the group with CCA. Because the control group only included 38 subjects, the reference trajectory for normal brain development was built using open-source template brains (Gholipour et al., 2017). In the future, efforts could be made towards increasing the sample size in order to extract more robust features and define our own reference trajectory.

\section{Conclusion}

In this work, we presented a novel shape analysis pipeline to characterize the anatomical variability of fetuses with abnormal corpus callosum. The tools we introduced here are promising for the depiction of healthy and impaired fetuses and can be generalized to any dataset of fetal brain MRIs. We have also highlighted the lack of robust, fully automated pipelines for both the preprocessing and analysis of fetal MRIs. In the future, we aim at bridging this gap by developing open-source pipelines that facilitate data preparation and analysis and include the shape analysis tools introduced here. Postnatal brain imaging has already benefited from such pipelines, such as the Clinica software (Routier et al., 2021), which encompasses a variety of analysis tools and several imaging modalities. We further intend to work on spatiotemporal models in order to construct trajectories describing the growth of fetuses with abnormalities, in the spirit of the recently published atlas of fetal brains with spina bifida (Fidon et al., 2021b).

\section{Acknowledgments}

This work was partly funded by the last author's chair in the PRAIRIE institute funded by the French national agency ANR as part of the "Investissements d'avenir" programme under the reference ANR-19- P3IA-0001.

\section{Ethical Standards}

This work follows appropriate ethical standards in conducting research and writing the manuscript, following all applicable laws and regulations regarding treatment of human subjects. This work has been granted ethics approval by the Institutional Review Board of the Comité d'éthique de la recherche en imagerie médicale (CERIUM) under the reference CRM-2112-215.

\section{Conflicts of Interest}


The authors declare no conflicts of interest.

\section{References}

Amir Alansary, Matthew Lee, Kevin Keraudren, Bernhard Kainz, Christina Malamateniou, Mary Rutherford, Joseph Hajnal, Ben Glocker, and Daniel Rueckert. Automatic brain localization in fetal mri using superpixel graphs. pages 13-22, 072015 . ISBN 3319279289. doi: 10.1007/978-3-319-27929-9_2.

Nickie Andescavage, Adre du Plessis, Robert McCarter, Ahmed Serag, Iordanis Evangelou, Gilbert Vezina, Richard Robertson, and Catherine Limperopoulos. Complex trajectories of brain development in the healthy human fetus. Cerebral cortex (New York, N.Y. : 1991), 27, 10 2016. doi: 10.1093/cercor/bhw306.

Jérémie Anquez, Elsa Angelini, and Isabelle Bloch. Automatic segmentation of head structures on fetal mri. pages 109 - 112, 08 2009. doi: 10.1109/ISBI.2009.5192995.

Audrey Bénézit, Lucie Hertz-Pannier, Ghislaine Dehaene-Lambertz, Karla Monzalvo, David Germanaud, Delphine Duclap, Pamela Guevara, Jean-François Mangin, Cyril Poupon, Marie-Laure Moutard, and Jessica Dubois. Organising white matter in a brain without corpus callosum fibres. Cortex, 63:155-171, February 2015. doi: 10.1016/j.cortex.2014. 08.022. URL https://hal .archives-ouvertes.fr/hal-02436280.

Oualid Benkarim, Gerard Sanroma, Veronika Zimmer, Emma Muñoz-Moreno, Nadine Hahner, Elisenda Eixarch, Oscar Camara, Miguel Ángel González Ballester, and Gemma Piella. Toward the automatic quantification of in utero brain development in $3 \mathrm{~d}$ structural mri: A review: Quantification of fetal brain development. Human Brain Mapping, 38, 02 2017. doi: 10.1002/hbm.23536.

Oualid Benkarim, Nadine Hahner, Gemma Piella, Eduard Gratacos, Miguel Ángel González Ballester, Elisenda Eixarch, and Gerard Sanroma. Cortical folding alterations in fetuses with isolated non-severe ventriculomegaly. NeuroImage: Clinical, 18:103-114, 01 2018. doi: 10.1016/j.nicl.2018.01.006.

Oualid Benkarim, Gemma Piella, Islem Rekik, Nadine Hahner, Elisenda Eixarch, Gang Li, Miguel Ángel González Ballester, and Gerard Sanroma. A novel approach to multiple anatomical shape analysis: Application to fetal ventriculomegaly. Medical Image Analysis, 64:101750, 06 2020. doi: 10.1016/j.media.2020.101750.

Anat Biegon and Chen Hoffmann. Quantitative magnetic resonance imaging of the fetal brain in utero: Methods and applications. World journal of radiology, 6:523-9, 082014. doi: 10.4329 /wjr.v6.i8.523.

Alexandre Bône, Maxime Louis, Benoît Martin, and Stanley Durrleman. Deformetrica 4: an open-source software for statistical shape analysis. In ShapeMI@ MICCAI 2018, Granada, Spain, September 2018. URL https://hal.inria.fr/hal-01874752. 
Gary Christensen, Richard Rabbitt, and Michael Miller. Deformable template using large deformation kinematics. IEEE transactions on image processing : a publication of the IEEE Signal Processing Society, 5:1435-47, 02 1996. doi: 10.1109/83.536892.

Cedric Clouchoux, Dimitri Kudelski, Ali Gholipour, Simon Warfield, Sophie Viseur, Marine Bouyssi-Kobar, Jean-Luc Mari, Alan Evans, Adre du Plessis, and Catherine Limperopoulos. Quantitative in vivo mri measurement of cortical development in the fetus. Brain structure function, 217:127-39, 05 2011. doi: 10.1007/s00429-011-0325-x.

Cedric Clouchoux, Adre du Plessis, Marine Bouyssi-Kobar, W Tworetzky, D.B. Mcelhinney, David Brown, Ali Gholipour, Dimitri Kudelski, Simon Warfield, Robert Mccarter, R.L. Robertson, Alan Evans, Jane Newburger, and Catherine Limperopoulos. Delayed cortical development in fetuses with complex congenital heart disease. Cerebral cortex (New York, N.Y. : 1991), 23, 09 2012. doi: 10.1093/cercor/bhs281.

J Corbett-Detig, Piotr Habas, Julia Scott, Kio Kim, Vidya Rajagopalan, Patrick Mcquillen, A Barkovich, O Glenn, and C Studholme. 3d global and regional patterns of human fetal subplate growth determined in utero. Brain structure function, 215:255-63, 10 2010. doi: $10.1007 / \mathrm{s} 00429-010-0286-5$.

Vianney Debavelaere, Stanley Durrleman, and Stéphanie Allassonnière. Learning the clustering of longitudinal shape data sets into a mixture of independent or branching trajectories. International Journal of Computer Vision, 128, 12 2020. doi: 10.1007/ s11263-020-01337-8.

Noémie Debroux, Carole Guyader, and Luminita Vese. Multiscale Registration, pages 115127. 04 2021. ISBN 978-3-030-75548-5. doi: 10.1007/978-3-030-75549-2_10.

Maria Deprez, Gerardine Quaghebeur, Mary Rutherford, Joseph Hajnal, and Julia Schnabel. Reconstruction of fetal brain mri with intensity matching and complete outlier removal. Medical image analysis, 16, 08 2012. doi: 10.1016/j.media.2012.07.004.

Vincent Des Portes, Anne Rolland, Juan Velazquez-Dominguez, Emeline Peyric, MariePierre Cordier, Pascal Gaucherand, Jérôme Massardier, Mona Massoud, Aurore Curie, Anne-Sophie Pellot, Francois Rivier, Audrey Lacalm, Amélie Clément, Dorothée Ville, and Laurent Guibaud. Outcome of isolated agenesis of the corpus callosum: A populationbased prospective study. European Journal of Paediatric Neurology, 22(1):82 - 92, January 2018. doi: 10.1016/j.ejpn.2017.08.003. URL https://hal.umontpellier.fr/ hal-01796396.

Stanley Durrleman, Stéphanie Allassonnière, and Sarang Joshi. Sparse adaptive parameterization of variability in image ensembles. International Journal of Computer Vision, 101:1-23, 11 2012. doi: 10.1007/s11263-012-0556-1.

Francesco D'Antonio, Giorgio Pagani, Alessandra Familiari, Asma Khalil, Tally-Lerman Sagies, Gustavo Malinger, Zvi Leibovitz, Catherine Garel, Marie Laure Moutard, Gianluigi Pilu, Amar Bhide, Ganesh Acharya, Martina Leombroni, Lamberto Manzoli, 
Aris Papageorghiou, and Federico Prefumo. Outcomes Associated With Isolated Agenesis of the Corpus Callosum: A Meta-analysis. 138(3), 09 2016. ISSN 0031-4005. doi: 10.1542/peds.2016-0445. URL https ://doi .org/10.1542/peds.2016-0445, e20160445.

Michael Ebner, Guotai Wang, Wenqi Li, Michael Aertsen, Premal Amrishkumar Patel, Rosalind Aughwane, Andrew Melbourne, Tom Doel, Steven Dymarkowski, Paolo De Coppi, Anna Louise David, Jan A Deprest, Sébastien Ourselin, and Tom Vercauteren. An automated framework for localization, segmentation and super-resolution reconstruction of fetal brain mri. Neuroimage, 206, 2020.

Lucas Fidon, Michael Aertsen, Nada Mufti, Thomas Deprest, Doaa Emam, Frédéric Guffens, Ernst Schwartz, Michael Ebner, Daniela Prayer, Gregor Kasprian, Anna David, Andrew Melbourne, Sébastien Ourselin, Jan Deprest, Georg Langs, and Tom Vercauteren. Distributionally robust segmentation of abnormal fetal brain 3d mri, 08 2021a.

Lucas Fidon, Elizabeth Viola, Nada Mufti, Anna David, Andrew Melbourne, Philippe Demaerel, Sébastien Ourselin, Tom Vercauteren, Jan Deprest, and Michael Aertsen. A spatio-temporal atlas of the developing fetal brain with spina bifida aperta. Open Research Europe, 1:123, 10 2021b. doi: 10.12688/openreseurope.13914.1.

Fleur Gaudfernau, Eleonore Blondiaux, and Stéphanie Allassonière. Analysis of the Anatomical Variability of Fetal Brains with Corpus Callosum Agenesis. In Carole H. Sudre, Roxane Licandro, Christian Baumgartner, Andrew Melbourne, Adrian Dalca, Jana Hutter, Ryutaro Tanno, Esra Abaci Turk, Koen Van Leemput, Jordina Torrents Barrena, William M. Wells, and Christopher Macgowan, editors, MICCAI 2021 - Perinatal, Preterm and Paediatric Image Analysis Workshop, volume 12959 of LNCS Lecture Notes in Computer Science, Strasbourg, France, October 2021. Springer. doi: 10.1007/978-3-030-87735-4\_26.

Ali Gholipour, Judy Estroff, and Simon Warfield. Robust super-resolution volume reconstruction from slice acquisitions: Application to fetal brain mri. IEEE transactions on medical imaging, 29:1739-58, 10 2010. doi: 10.1109/TMI.2010.2051680.

Ali Gholipour, Judy Estroff, Carol Barnewolt, Susan Connolly, and Simon Warfield. Fetal brain volumetry through mri volumetric reconstruction and segmentation. International journal of computer assisted radiology and surgery, 6:329-39, 05 2011. doi: 10.1007/ s11548-010-0512-x.

Ali Gholipour, Alireza Akhondi-Asl, Judy Estroff, and Simon Warfield. Multi-atlas multishape segmentation of fetal brain mri for volumetric and morphometric analysis of ventriculomegaly. NeuroImage, 60:1819-31, 04 2012. doi: 10.1016/j.neuroimage.2012.01.128.

Ali Gholipour, Judy Estroff, Carol Barnewolt, Richard Robertson, Patricia Grant, Borjan Gagoski, Simon Warfield, Onur Afacan, Susan Connolly, Jeffrey Neil, Adam Wolfberg, and Robert Mulkern. Fetal mri: A technical update with educational aspirations. Concepts in Magnetic Resonance Part A, 43, 03 2015. doi: 10.1002/cmr.a.21321. 
Ali Gholipour et al. A normative spatiotemporal mri atlas of the fetal brain for automatic segmentation and analysis of early brain growth. Scientific Reports, 7, 12 2017. doi: 10.1038/s41598-017-00525-w.

S. Glatter, G. Kasprian, D. Bettelheim, B. Ulm, M. Weber, R. Seidl, D. Prayer, and M.C. Diogo. Beyond isolated and associated: A novel fetal $\mathrm{mr}$ imaging-based scoring system helps in the prenatal prognostication of callosal agenesis. American Journal of Neuroradiology, 42(4):782-786, 2021. ISSN 0195-6108. doi: 10.3174/ajnr.A7064. URL http://www . ajnr.org/content/42/4/782.

Piotr Habas, Kio Kim, James Corbett-Detig, François Rousseau, Orit Glenn, A Barkovich, and Colin Studholme. A spatiotemporal atlas of mr intensity, tissue probability and shape of the fetal brain with application to segmentation. NeuroImage, 53:460-70, 11 2010. doi: 10.1016/j.neuroimage.2010.06.054.

Piotr Habas, Julia Scott, Ahmad Roosta, Vidya Rajagopalan, Kio Kim, François Rousseau, A Barkovich, Orit Glenn, and Colin Studholme. Early folding patterns and asymmetries of the normal human brain detected from in utero mri. Cerebral cortex (New York, N.Y. : 1991), 22:13-25, 05 2011. doi: 10.1093/cercor/bhr053.

Benjamin Hou, Bishesh Khanal, Amir Alansary, Steven McDonagh, Alice Davidson, Mary Rutherford, Jo Hajnal, Daniel Rueckert, Ben Glocker, and Bernhard Kainz. 3-d reconstruction in canonical co-ordinate space from arbitrarily oriented 2-d images. IEEE Transactions on Medical Imaging, PP, 09 2017. doi: 10.1109/TMI.2018.2798801.

Hui-Hsin Hu, Chih-I Hung, Yu-Te Wu, Hui-Yun Chen, Jen-Chuen Hsieh, and Wan-Yuo Guo. Regional quantification of developing human cortical shape with a three-dimensional surface-based magnetic resonance imaging analysis in utero. The European journal of neuroscience, 34:1310-9, 10 2011. doi: 10.1111/j.1460-9568.2011.07855.x.

Mark Ison, Eva Weigl, René Donner, Gregor Kasprian, Daniela Prayer, and Georg Langs. Fully automated brain extraction and orientation in raw fetal mri. 10 2012. doi: 10. 13140/2.1.2312.8966.

Francois Jacob, Piotr Habas, Kio Kim, James Corbett-Detig, Duan Xu, Colin Studholme, and Orit Glenn. Fetal hippocampal development: Analysis by magnetic resonance imaging volumetry. Pediatric research, 69:425-9, 05 2011. doi: 10.1203/PDR. 0b013e318211dd7f.

András Jakab, Gregor Kasprian, Ernst Schwartz, Gerlinde Maria Gruber, Christian Mitter, Daniela Prayer, Veronika Schöpf, and Georg Langs. Disrupted developmental organization of the structural connectome in fetuses with corpus callosum agenesis. NeuroImage, 02 2015. doi: 10.1016/j.neuroimage.2015.02.038.

Shuzhou Jiang, Hui Xue, Alan Glover, Mary Rutherford, Daniel Rueckert, and Joseph Hajnal. Mri of moving subjects using multislice snapshot images with volume reconstruction (svr): Application to fetal, neonatal, and adult brain studies. IEEE transactions on medical imaging, 26:967-80, 08 2007. doi: 10.1109/TMI.2007.895456. 
Bernhard Kainz, Kevin Keraudren, Vanessa Kyriakopoulou, Mary Rutherford, Joseph Hajnal, and Daniel Rueckert. Fast fully automatic brain detection in fetal mri using dense rotation invariant image descriptors. 04 2014. doi: 10.1109/ISBI.2014.6868098.

Bernhard Kainz, Markus Steinberger, Wolfgang Wein, Maria Deprez, Christina Malamateniou, Kevin Keraudren, Thomas Torsney-Weir, Mary Rutherford, Paul Aljabar, Joseph Hajnal, and Daniel Rueckert. Fast volume reconstruction from motion corrupted stacks of $2 \mathrm{~d}$ slices. IEEE Transactions on Medical Imaging, 34, 03 2015. doi: 10.1109/TMI.2015.2415453.

Gregor Kasprian, Peter Brugger, Veronika Schöpf, Christian Mitter, Michael Weber, Johannes Hainfellner, and Daniela Prayer. Assessing prenatal white matter connectivity in commissural agenesis. Brain : a journal of neurology, 136:168-79, 01 2013. doi: 10.1093/brain/aws332.

Kevin Keraudren, Vanessa Kyriakopoulou, Mary Rutherford, Joseph Hajnal, and Daniel Rueckert. Localisation of the brain in fetal mri using bundled sift features. volume 16, pages 582-9, 09 2013. ISBN 978-3-642-38708-1. doi: 10.1007/978-3-642-40811-3_73.

Kevin Keraudren, Maria Deprez, Vanessa Kyriakopoulou, Christina Malamateniou, Mary Rutherford, Bernhard Kainz, Joseph Hajnal, and D. Rueckert. Automated fetal brain segmentation from 2d mri slices for motion correction. NeuroImage, pages -, 072014. doi: 10.1016/j.neuroimage.2014.07.023.

Nadieh Khalili, Pim Moeskops, Nathalie Claessens, S. Scherpenzeel, Elise Turk, R. Heus, Manon Benders, M. Viergever, J. Pluim, and Ivana Isgum. Automatic segmentation of the intracranial volume in fetal $\mathrm{mr}$ images. pages 42-51, 09 2017. ISBN 978-3-319-67560-2. doi: 10.1007/978-3-319-67561-9_5.

Kio Kim, Piotr Habas, François Rousseau, Orit Glenn, Anthony Barkovich, and Colin Studholme. Intersection based motion correction of multislice mri for 3-d in utero fetal brain image formation. IEEE transactions on medical imaging, 29:146-58, 10 2009. doi: 10.1109/TMI.2009.2030679.

Vinka Knezović et al. Underdevelopment of the human hippocampus in callosal agenesis: An in vivo fetal mri study. American Journal of Neuroradiology, 40, 02 2019. doi: 10.3174/ajnr.A5986.

Vanessa Kyriakopoulou, Deniz Vatansever, Samia Elkommos, Sarah Dawson, Amy Mcguinness, Joanna Allsop, Zoltán Molnár, Joseph Hajnal, and Mary Rutherford. Cortical overgrowth in fetuses with isolated ventriculomegaly. Cerebral cortex (New York, N.Y. : 1991), 24, 03 2013. doi: 10.1093/cercor/bht062.

Vanessa Kyriakopoulou, Deniz Vatansever, Alice Davidson, Prachi Patkee, Samia Elkommos, Andrew Chew, Miriam Martinez-Biarge, Bibbi Hagberg, Mellisa Damodaram, Joanna Allsop, Matt Fox, Joseph Hajnal, and Mary Rutherford. Normative biometry of the fetal brain using magnetic resonance imaging. Brain Structure and Function, 222, 07 2017. doi: 10.1007/s00429-016-1342-6. 
Martina Leombroni, Asma Khalil, Marco Liberati, and Francesco dAntonio. Fetal midline anomalies: Diagnosis and counselling part 1: Corpus callosum anomalies. European journal of paediatric neurology : EJPN : official journal of the European Paediatric Neurology Society, 22 6:951-962, 2018.

Haotian Li, Guohui Yan, Wanrong Luo, Tingting Liu, Yan Wang, Ruibin Liu, Weihao Zheng, Yi Zhang, Kui Li, Li Zhao, Catherine Limperopoulos, Yu Zou, and Dan Wu. Mapping fetal brain development based on automated segmentation and $4 \mathrm{~d}$ brain atlasing. Brain Structure and Function, 226, 07 2021. doi: 10.1007/s00429-021-02303-x.

Maxime Louis, Alexandre Bône, Benjamin Charlier, and Stanley Durrleman. Parallel transport in shape analysis: a scalable numerical scheme. 11 2017. doi: 10.1007/ 978-3-319-68445-1_4.

Steven McDonagh, Benjamin Hou, Konstantinos Kamnitsas, Ozan Oktay, Amir Alansary, and Bernhard Kainz. Context-sensitive super-resolution for fast fetal magnetic resonance imaging. 022017.

Michael Miller, Alain Trouvé, and Laurent Younes. On the metrics and euler-lagrange equations of computational anatomy. Annual review of biomedical engineering, 4:375405, 02 2002. doi: 10.1146/annurev.bioeng.4.092101.125733.

Michael Miller, Alain Trouvé, and Laurent Younes. Geodesic shooting for computational anatomy. Journal of mathematical imaging and vision, 24:209-228, 01 2006. doi: 10. 1007/s10851-005-3624-0.

Y Nakata, A.J. Barkovich, M Wahl, Z Strominger, Rita Jeremy, M Wakahiro, Pratik Mukherjee, and Elliott Sherr. Diffusion abnormalities and reduced volume of the ventral cingulum bundle in agenesis of the corpus callosum: A 3t imaging study. AJNR. American journal of neuroradiology, 30:1142-8, 02 2009. doi: 10.3174/ajnr.A1527.

Kelly Payette, Ueli Moehrlen, Luca Mazzone, Nicole Ochsenbein-Kölble, Ruth Tuura, Raimund Kottke, Martin Meuli, and András Jakab. Longitudinal Analysis of Fetal MRI in Patients with Prenatal Spina Bifida Repair, pages 161-170. 10 2019. ISBN 978-3-03032874-0. doi: 10.1007/978-3-030-32875-7_18.

Julien Pontabry, François Rousseau, Colin Studholme, M. Koob, and J.-L Dietemann. A discriminative feature selection approach for shape analysis: application to fetal brain cortical folding study. Medical Image Analysis, 35, 01 2015. doi: 10.1016/j.media.2016. 07.005.

A. Qiu, L. Younes, M. I. Miller, and J. G. Csernansky. Parallel transport in diffeomorphisms distinguishes the time-dependent pattern of hippocampal surface deformation due to healthy aging and the dementia of the alzheimer's type. NeuroImage, 40:68-76, 042008. doi: 10.1016/j.neuroimage.2007.11.041.

Vidya Rajagopalan, Julia Scott, Piotr Habas, Kio Kim, James Corbett-Detig, François Rousseau, A Barkovich, Orit Glenn, and Colin Studholme. Local tissue growth patterns underlying normal fetal human brain gyrification quantified in utero. The Journal of 
neuroscience : the official journal of the Society for Neuroscience, 31:2878-87, 022011. doi: 10.1523/JNEUROSCI.5458-10.2011.

Vidya Rajagopalan, Julia Scott, Piotr Habas, Kio Kim, François Rousseau, Orit Glenn, A Barkovich, and Colin Studholme. Mapping directionality specific volume changes using tensor based morphometry: An application to the study of gyrogenesis and lateralization of the human fetal brain. NeuroImage, 63:947-58, 04 2012. doi: 10.1016/j.neuroimage. 2012.03.092.

Vidya Rajagopalan, Jodie Votava-Smith, Xiaowei Zhuang, Jessica Brian, Lila Marshall, Ashok Panigrahy, and Lisa Paquette. Fetuses with single ventricle congenital heart disease manifest impairment of regional brain growth. Prenatal Diagnosis, 38, 10 2018. doi: $10.1002 / \mathrm{pd} .5374$.

Vidya Rajagopalan, Sean Deoni, Ashok Panigrahy, and Moriah Thomason. Is fetal mri ready for neuroimaging prime time? an examination of progress and remaining areas for development. Developmental Cognitive Neuroscience, 51:100999, 08 2021. doi: 10.1016/ j.den.2021.100999.

Martin Rajchl, Matthew Lee, Ozan Oktay, Konstantinos Kamnitsas, Jonathan PasseratPalmbach, Wenjia Bai, Bernhard Kainz, and Daniel Rueckert. Deepcut: Object segmentation from bounding box annotations using convolutional neural networks. IEEE Transactions on Medical Imaging, PP, 05 2016. doi: 10.1109/TMI.2016.2621185.

Charles Raybaud. The corpus callosum, the other great forebrain commissures, and the septum pellucidum: Anatomy, development, and malformation. Neuroradiology, 52:44777, 06 2010. doi: 10.1007/s00234-010-0696-3.

Caitlin Rollins, Cynthia Ortinau, Christian Stopp, Kevin Friedman, Wayne Tworetzky, Borjan Gagoski, Clemente Velasco-Annis, Onur Afacan, Lana Vasung, Jeanette Beaute, Valerie Rofeberg, Judy Estroff, Patricia Grant, Janet Soul, Edward Yang, David Wypij, Ali Gholipour, Simon Warfield, and Jane Newburger. Regional brain growth trajectories in fetuses with congenital heart disease. Annals of Neurology, 89, 11 2020. doi: 10.1002/ ana.25940.

François Rousseau, Colin Studholme, Renaud Jardri, and Moriah E. Thomason. In Vivo Human Fetal Brain Analysis Using MR Imaging, pages 407-427. Springer International Publishing, Cham, 2016. ISBN 978-3-319-22023-9. doi: 10.1007/978-3-319-22023-9_20.

François Rousseau, Orit Glenn, Bistra Iordanova, Claudia Rodriguez-Carranza, Daniel Vigneron, James Barkovich, and Colin Studholme. Registration-based approach for reconstruction of high-resolution in utero fetal $\mathrm{mr}$ brain images. Academic radiology, 13: 1072-81, 10 2006. doi: 10.1016/j.acra.2006.05.003.

François Rousseau, Kio Kim, C Studholme, M. Koob, and J Dietemann. On super-resolution for fetal brain mri. volume 13, pages 355-62, 09 2010. ISBN 978-3-642-15744-8. doi: 10.1007/978-3-642-15745-5_44. 
François Rousseau, Estanislao Oubel, Julien Pontabry, Marc Schweitzer, Colin Studholme, M. Koob, and Jean-Louis Dietemann. Btk: An open-source toolkit for fetal brain mr image processing. Computer methods and programs in biomedicine, 109, 10 2012. doi: 10.1016/j.cmpb.2012.08.007.

Alexandre Routier, Ninon Burgos, Mauricio Díaz, Michael Bacci, Simona Bottani, Omar El-Rifai, Sabrina Fontanella, Pietro Gori, Jérémy Guillon, Alexis Guyot, Ravi Hassanaly, Thomas Jacquemont, Pascal Lu, Arnaud Marcoux, Tristan Moreau, Jorge SamperGonzalez, Marc Teichmann, Elina Thibeau-Sutre, Ghislain Vaillant, and Olivier Colliot. Clinica: An open-source software platform for reproducible clinical neuroscience studies. Frontiers in Neuroinformatics, 15, 08 2021. doi: 10.3389/fninf.2021.689675.

Seyed Sadegh Salehi, Seyed Raein Hashemi, Clemente Velasco-Annis, Abdelhakim Ouaalam, Judy Estroff, Deniz Erdogmus, Simon Warfield, and Ali Gholipour. Real-time automatic fetal brain extraction in fetal mri by deep learning. 04 2018. doi: 10.1109/ISBI.2018. 8363675 .

Susana Ferreira Santo, Francesco dAntonio, Tessa Homfray, Philip Rich, Gianluigi Pilu, Amar Bhide, Baskaran Thilaganathan, and Aris T. Papageorghiou. Counseling in fetal medicine: agenesis of the corpus callosum. Ultrasound in Obstetrics $\&$ Gynecology, 40, 2012 .

Ernst Schwartz, Mariana Cardoso Diogo, Sarah Glatter, Rainer Seidl, Peter C Brugger, Gerlinde M Gruber, Herbert Kiss, Karl-Heinz Nenning, Georg Langs, Daniela Prayer, and Gregor Kasprian and. The prenatal morphomechanic impact of agenesis of the corpus callosum on human brain structure and asymmetry. Cerebral Cortex, apr 2021. doi: 10.1093/cercor/bhab066. URL https://doi.org/10.1093\%2Fcercor\%2Fbhab066.

Julia Scott, Piotr Habas, Kio Kim, Vidya Rajagopalan, Kia Hamzelou, James CorbettDetig, A Barkovich, Orit Glenn, and Colin Studholme. Growth trajectories of the human fetal brain tissues estimated from $3 \mathrm{~d}$ reconstructed in utero mri. International journal of developmental neuroscience : the official journal of the International Society for Developmental Neuroscience, 29:529-36, 08 2011a. doi: 10.1016/j.ijdevneu.2011.04.001.

Julia Scott, Kia Hamzelou, Vidya Rajagopalan, Piotr Habas, Kio Kim, A Barkovich, Orit Glenn, and Colin Studholme. 3d morphometric analysis of human fetal cerebellar development. Cerebellum (London, England), 11:761-70, 12 2011b. doi: 10.1007/ s12311-011-0338-2.

Julia Scott, Piotr Habas, Vidya Rajagopalan, Kio Kim, A Barkovich, Orit Glenn, and Colin Studholme. Volumetric and surface-based 3d mri analyses of fetal isolated mild ventriculomegaly. Brain structure function, 218, 05 2012. doi: 10.1007/s00429-012-0418-1.

Colin Studholme and François Rousseau. Quantifying and modelling tissue maturation in the living human fetal brain. International journal of developmental neuroscience : the official journal of the International Society for Developmental Neuroscience, 32, 072013. doi: 10.1016/j.ijdevneu.2013.06.006. 
Youssef Taleb, Marc Schweitzer, Colin Studholme, M. Koob, Jean-Louis Dietemann, and François Rousseau. Automatic template-based brain extraction in fetal $\mathrm{mr}$ images. 06 2013.

Tomo Tarui, Neel Madan, Nabgha Farhat, Rie Kitano, Asye Ceren Tanritanir, George Graham, Borjan Gagoski, Alexa Craig, Caitlin K Rollins, Cynthia Ortinau, Vidya Iyer, Rudolph Pienaar, Diana W Bianchi, P Ellen Grant, and Kiho Im. Disorganized patterns of sulcal position in fetal brains with agenesis of corpus callosum. Cereb Cortex, 28(9): 3192-3203, 20180901 2018. ISSN 1460-2199. doi: 10.1093/cercor/bhx191.

Sébastien Tourbier, Xavier Bresson, Patric Hagmann, Jean-Philippe Thiran, Reto Meuli, and Meritxell Bach Cuadra. An efficient total variation algorithm for super-resolution in fetal brain mri with adaptive regularization. NeuroImage, 118, 06 2015a. doi: 10.1016/j. neuroimage.2015.06.018.

Sébastien Tourbier, Patric Hagmann, Maud Cagneaux, Laurent Guibaud, Subrahmanyam Gorthi, Marie Schaer, Jean-Philippe Thiran, Reto Meuli, and Meritxell Bach Cuadra. Automatic brain extraction in fetal mri using multi-atlas-based segmentation. volume 9413, 02 2015b. doi: 10.1117/12.2081777.

Sébastien Tourbier, Clemente Velasco-Annis, Vahid Taimouri, Patric Hagmann, Reto Meuli, Simon Warfield, Meritxell Bach Cuadra, and Ali Gholipour. Automated template-based brain localization and extraction for fetal brain mri reconstruction. NeuroImage, 155, 04 2017. doi: 10.1016/j.neuroimage.2017.04.004.

Alain Trouvé. Diffeomorphisms groups and pattern matching in image analysis. International Journal of Computer Vision, 28(3):213-221, 1998. URL https://doi.org/10. 1023/A : 1008001603737 .

Daniel Warren, Daniel Connolly, and P Griffiths. Assessment of sulcation of the fetal brain in cases of isolated agenesis of the corpus callosum using in utero mr imaging. AJNR. American journal of neuroradiology, 31:1085-90, 06 2010. doi: 10.3174/ajnr.A1982.

R Wright, Vanessa Kyriakopoulou, Christian Ledig, Mary Rutherford, Joseph Hajnal, D Rueckert, and P Aljabar. Automatic quantification of normal cortical folding patterns from fetal brain mri. NeuroImage, 91, 01 2014. doi: 10.1016/j.neuroimage.2014.01.034.

R Wright, Antonios Makropoulos, Vanessa Kyriakopoulou, Prachi Patkee, Lisa Koch, Mary Rutherford, Joseph Hajnal, D Rueckert, and P Aljabar. Construction of a fetal spatiotemporal cortical surface atlas from in utero mri: Application of spectral surface matching. NeuroImage, 120, 06 2015. doi: 10.1016/j.neuroimage.2015.05.087.

Jue Wu, S.P. Awate, Daniel Licht, Cedric Clouchoux, Adre du Plessis, B.B. Avants, Arastoo Vossough, James Gee, and Catherine Limperopoulos. Assessment of mri-based automated fetal cerebral cortical folding measures in prediction of gestational age in the third trimester. AJNR. American journal of neuroradiology, 36, 062015. doi: 10.3174/ajnr.A4357. 
Analysis of the Anatomical Variability of Fetal Brains with Corpus Callosum Agenesis

Hyuk Jin Yun, Ai Wern Chung, Lana Vasung, Edward Yang, Tomo Tarui, Caitlin Rollins, Cynthia Ortinau, Patricia Grant, and Kiho Im. Automatic labeling of cortical sulci for the human fetal brain based on spatio-temporal information of gyrification. NeuroImage, 188, 12 2018. doi: 10.1016/j.neuroimage.2018.12.023.

Paul Yushkevich, Yang Gao, and Guido Gerig. Itk-snap: An interactive tool for semiautomatic segmentation of multi-modality biomedical images. volume 2016, pages 3342 3345, 08 2016. doi: 10.1109/EMBC.2016.7591443. 


\section{Appendix A. First mode of deformation.}

Fig. 9 presents the first mode of deformation, which was not retained during forward feature selection. Healthy subjects are more likely to score lower on the related component than subjects with CCA, though their distributions are closer to that of component 2 .

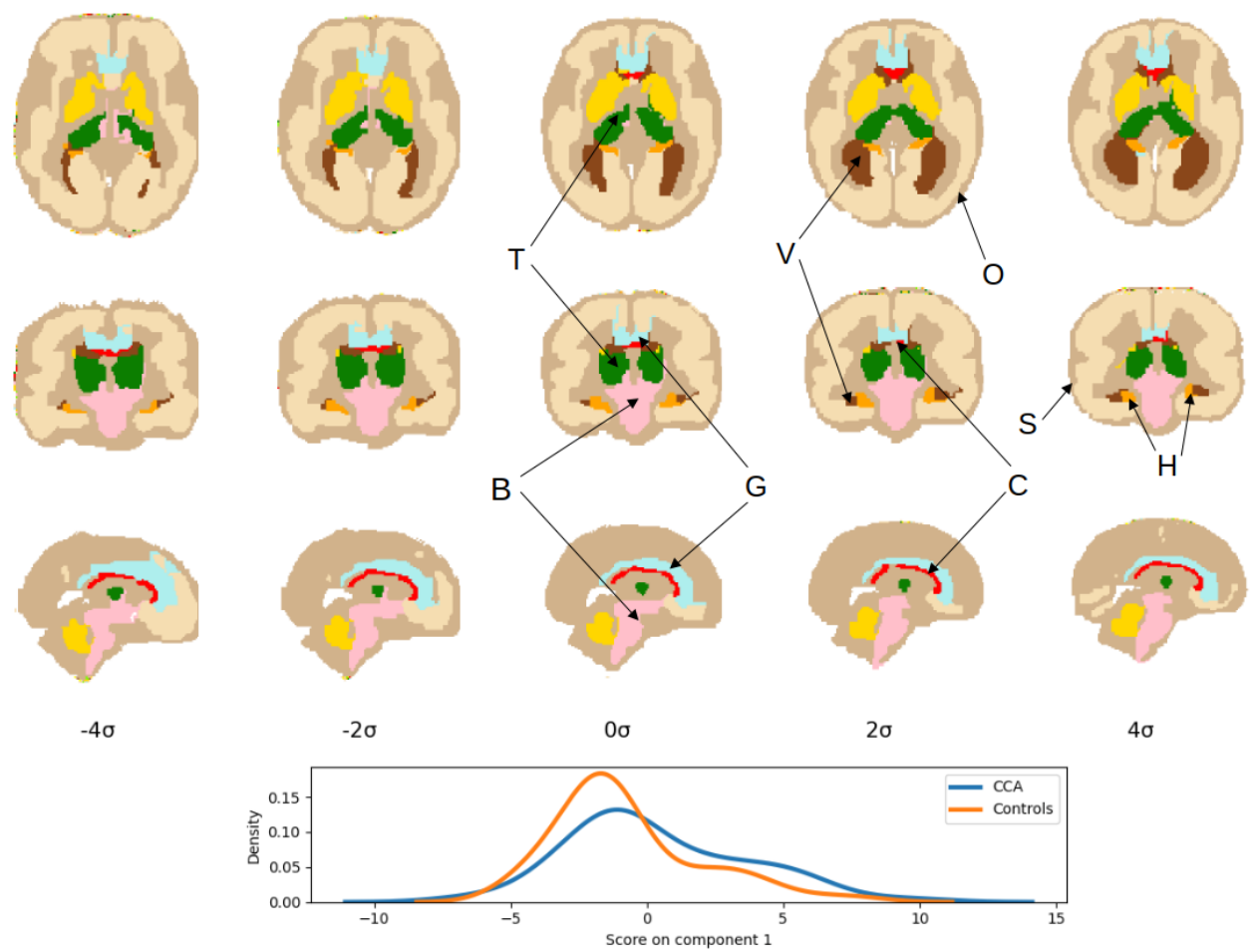

Figure 9: First mode of deformation applied to the segmentation of the template brain at age $31 \mathrm{GW}$. Top three rows: axial, coronal and sagittal views. Leftmost columns: geodesic shooting of the template brain by the first mode of deformation at $-4 \sigma$ and $-2 \sigma$. Central column: template brain. Rightmost columns: geodesic shooting of the template brain by the first mode of deformation at $+2 \sigma$ and $+4 \sigma$. Bottom row: distribution of the subjects scores on component 1. B: brainstem. C: corpus callosum. G: cingulate gyrus. H: hippocampi. O: occipital cortex. S: superior temporal sulcus. T: thalami. V: lateral ventricles. 